\title{
HIGHER INTEGRABILITY FOR THE SINGULAR POROUS MEDIUM SYSTEM
}

\author{
VERENA BÖGELEIN, FRANK DUZAAR, AND CHRISTOPH SCHEVEN
}

\begin{abstract}
In this paper we establish in the fast diffusion range the higher integrability of the spatial gradient of weak solutions to porous medium systems. The result comes along with an explicit reverse Hölder inequality for the gradient. The novel feature in the proof is a suitable intrinsic scaling for space-time cylinders combined with reverse Hölder inequalities and a Vitali covering argument within this geometry. The main result holds for the natural range of parameters suggested by other regularity results. Our result applies to general fast diffusion systems and includes both, nonnegative and signed solutions in the case of equations. The methods of proof are purely vectorial in their structure.
\end{abstract}

\section{INTRODUCTION AND RESULTS}

In this paper we study regularity of solutions to second-order parabolic systems

$$
\partial_{t} u-\operatorname{div} \mathbf{A}\left(x, t, u, D\left(|u|^{m-1} u\right)\right)=\operatorname{div} F
$$

on a space-time cylinder $\Omega_{T}:=\Omega \times(0, T)$ over a bounded domain $\Omega \subset \mathbb{R}^{n}, n \in \mathbb{N}$, and $T>0$. Precise structural assumptions for the vector field $\mathbf{A}$ are presented later. The principal prototype is the inhomogeneous porous medium system

$$
\partial_{t} u-\Delta\left(|u|^{m-1} u\right)=\operatorname{div} F,
$$

with $m>0$. As usual, solutions to (1.1) are taken in a weak sense, i.e. they are assumed to belong to a parabolic Sobolev space whose amount of integrability is determined by the growth of the vector field $\mathbf{A}$ with respect to the gradient variable, cf. Definition 2.1. With the choice $m=1$ we recover the heat equation. Equation (1.2) has a different behavior when $m>1$ or $m<1$. The first case is called slow diffusion range, since disturbances propagate with finite speed and free boundaries may occur, while in the second case disturbances propagate with infinite speed and extinction in finite time may occur. This range is called fast diffusion range. For more information on the theory for the porous medium equation and related regularity results we refer to $[5,7,20,21]$ and the references therein.

The main purpose of this paper is to establish a higher integrability result for the gradient of weak solutions of porous medium equations and systems of the type (1.1) in the fast diffusion range. More precisely, we show that there exists a universal constant $\varepsilon>0$, such that

$$
D\left(|u|^{m-1} u\right) \in L_{\mathrm{loc}}^{2+\varepsilon},
$$

whenever $u$ is a weak solution to (1.1), thereby ensuring that for weak solutions $u$ of the porous medium system the spatial gradient of $|u|^{m-1} u$ belongs to a slightly better Lebesgue space than the natural energy space $L^{2}$. This implies that porous medium systems as in (1.1) possess the self-improving property of integrability. Our result comes along with a quantitative local reverse Hölder type estimate for $\left|D\left(|u|^{m-1} u\right)\right|$; see Theorem 2.2. The higher integrability for porous medium systems as in (1.1) has been an open

Date: November 26, 2018.

2010 Mathematics Subject Classification. 35B65, 35K67, 35K40, 35K55.

Key words and phrases. Porous medium type systems, higher integrability, gradient estimates. 
problem for a long time, even in the case of equations and non-negative solutions. Here we give a positive answer in the fast diffusion range

$$
m_{c}:=\frac{(n-2)_{+}}{n+2}<m \leq 1 .
$$

The lower bound on $m$ is natural and appears also in other regularity results for porous medium equations, cf. the discussion in $[8, \S 6.21]$. For example, solutions might be unbounded in the super-critical range $0<m \leq m_{c}$.

The central idea in the proof of our main result is a new kind of intrinsic geometry. Until now, variants of this idea have been successfully used in establishing the self-improving property of integrability for the parabolic $p$-Laplacian system [16] and very recently in the slow diffusion range $m \geq 1$ for the porous medium equation [11] and system [3]. The central idea here is the construction of suitable intrinsic cylinders $Q_{r, s}\left(z_{o}\right):=B_{r}\left(x_{o}\right) \times$ $\left(t_{o}-s, t_{o}+s\right)$ with $z_{o}=\left(x_{o}, t_{o}\right)$. Since the equation is nonlinear with respect to $u$, we use cylinders whose space-time scaling depends on the mean values of $|u|^{1+m}$. This choice is dictated by the leading term on the right-hand side in the energy estimate, which is of order $1+m$; cf. Lemma 4.1. This heuristic argument motivates to consider space-time cylinders $Q_{r, s}\left(z_{o}\right)$, such that the quotient $\frac{s}{r^{\frac{1+m}{m}}}$ satisfies

$$
\frac{s}{r^{\frac{1+m}{m}}}=\theta^{1-m}, \text { with } \theta^{1+m} \approx \iint_{Q_{r, s}\left(z_{o}\right)} \frac{|u|^{1+m}}{r^{\frac{1+m}{m}}} \mathrm{~d} x \mathrm{~d} t .
$$

In this geometry the only ingredients for the proof of parabolic Sobolev-Poincaré and reverse Hölder type inequalities are the standard energy estimate and a gluing lemma; see Section 4. The construction of a system of such intrinsic cylinders is quite involved, since the cylinders on the right-hand side (1.5) also depend on the parameter $\theta$. In fact, we have to distinguish between two regimes, the non-singular and the singular regime. The first is characterized by the fact that cylinders are intrinsic, the latter by the fact that cylinders are only sub-intrinsic, which means that $(1.5)_{2}$ only holds as an inequality where the mean value integral is bounded from above by $\theta^{1+m}$. In both regimes we need to establish reverse Hölder type inequalities. In the actual construction of the cylinders, we modify the argument from [11]; see also [3] which is better suited to our purposes here.

At this stage some words to classify our result in the history of the problem of higher integrability are appropriate. In the stationary case of elliptic systems the self-improving property was first observed by Elcrat \& Meyers [17], see also the monographs [13, Chapter V, Theorem 2.1] and [15, Section 6.4] and the references therein. The first higher integrability result for parabolic systems goes back to Giaquinta \& Struwe [14, Theorem 2.1]. For parabolic systems with $p$-growth, whose principle prototype is the parabolic $p$-Laplacian system, the higher integrability of the gradient of weak solutions was established by Kinnunen \& Lewis [16] in the range $p>\frac{2 n}{n+2}$. This lower bound is natural and appears also in other contexts in the regularity theory of parabolic $p$-Laplace type systems; cf. the monograph [6]. In the meantime the result has been generalized in various directions, such as global results and higher order parabolic systems with $p$-growth; see $[1,4,18]$. The corresponding problem for the porous medium equation turned out to be more involved and remained open for a long time, even in the scalar case for non-negative solutions. Additionally to the obvious anisotropic behavior of the equation with respect to scalar multiplication of solutions, it is also not possible to add constants to a solution without destroying the property of being a solution. This difficulty has recently been overcome by Gianazza \& Schwarzacher [11] who proved in the slow diffusion range $m \geq 1$ that nonnegative weak solutions of (1.1) admit the self-improving property of higher integrability of the gradient. The main novelty in their proof is the use of a new intrinsic scaling. Instead of scaling cylinders with respect to $|D u|$ as in the case of the parabolic $p$-Laplacian (cf. [6] and the references therein), they work with cylinders which are intrinsically scaled with respect to $u$. The proof, however, uses the method of expansion of positivity and therefore 
can not be extended to signed solutions, porous medium type systems and the fast diffusion range. A simpler and more flexible proof, which does not rely on the expansion of positivity and which covers both signed solutions and porous medium systems is given in [3]. Finally, in [2] the higher integrability is shown for doubly nonlinear parabolic systems, whose prototype is

$$
\partial_{t}\left(|u|^{p-2} u\right)-\operatorname{div}\left(|D u|^{p-2} D u\right)=\operatorname{div}\left(|F|^{p-2} F\right) .
$$

In this equation aspects of both the porous medium equation and the parabolic $p$-Laplace equation play a role. Therefore the intrinsic scaling has to take into account the degeneracy of the system both with respect to the gradient variable and with respect to the solution itself. In [2] the higher integrability is established for exponents $p$ in the somewhat unexpected range $\max \left\{\frac{2 n}{n+2}, 1\right\}<p<\frac{2 n}{(n-2)_{+}}$. The lower bound also appears for the parabolic $p$-Laplace system [16], while the upper bound corresponds exactly to the lower bound in (1.4) for the porous medium equation in the fast diffusion range.

We point out that independently of us, Gianazza \& Schwarzacher [12] proved the higher integrability result in the scalar case for nonnegative solutions in the fast diffusion range (1.4). In contrast to [12], we prove the higher integrability regardless of whether the solution is non-negative or signed in the scalar case, or vector-valued in the case of systems. In another point, our results are also different. Instead of an inhomogeneity given by a bounded function $f$, we consider a right-hand side in divergence form $\operatorname{div} F$ with $F \in L^{\sigma}$ for some $\sigma>2$. In [12] the boundedness assumption on $f$ is imposed to ensure that weak solutions are bounded. Here, we are able to deal with unbounded solutions.

\section{NOTATION AND MAIN RESULT}

2.1. Notations. To keep formulations as simple as possible, we define the power of a vector or of a possibly negative number by

$$
\boldsymbol{u}^{\alpha}:=|u|^{\alpha-1} u, \quad \text { for } u \in \mathbb{R}^{N} \text { and } \alpha>0,
$$

which in the case $u=0$ and $\alpha \in(0,1)$ we interpret as $\boldsymbol{u}^{\alpha}=0$. Throughout the paper we write $z_{o}=\left(x_{o}, t_{o}\right) \in \mathbb{R}^{n} \times \mathbb{R}$ for points in space-time. We use space-time cylinders

$$
Q_{\varrho}^{(\theta)}\left(z_{o}\right):=B_{\varrho}^{(\theta)}\left(x_{o}\right) \times \Lambda_{\varrho}\left(t_{o}\right),
$$

where

and

$$
B_{\varrho}^{(\theta)}\left(x_{o}\right):=\left\{x \in \mathbb{R}^{n}:\left|x-x_{o}\right|<\theta^{\frac{m(m-1)}{1+m}} \varrho\right\}
$$

$$
\Lambda_{\varrho}\left(t_{o}\right):=\left(t_{o}-\varrho^{\frac{1+m}{m}}, t_{o}+\varrho^{\frac{1+m}{m}}\right)
$$

with some scaling parameter $\theta>0$. In the case $\theta=1$, we simply omit the parameter in the notation and write

$$
Q_{\varrho}\left(z_{o}\right):=B_{\varrho}\left(x_{o}\right) \times\left(t_{o}-\varrho^{\frac{1+m}{m}}, t_{o}+\varrho^{\frac{1+m}{m}}\right)
$$

instead of $Q_{\varrho}^{(1)}\left(z_{o}\right)$. If the center $z_{o}$ is clear from the context we omit it in the notation.

For a map $u \in L^{1}\left(0, T ; L^{1}\left(\Omega, \mathbb{R}^{N}\right)\right)$ and given measurable sets $A \subset \Omega$ and $E \subset \Omega_{T}$ with positive Lebesgue measure the slicewise mean $\langle u\rangle_{A}:(0, T) \rightarrow \mathbb{R}^{N}$ of $u$ on $A$ is defined by

$$
\langle u\rangle_{A}(t):=f_{A} u(t) \mathrm{d} x, \quad \text { for a.e. } t \in(0, T),
$$

whereas the mean value $(u)_{E} \in \mathbb{R}^{N}$ of $u$ on $E$ is defined by

$$
(u)_{E}:=\int_{E} u \mathrm{~d} x \mathrm{~d} t .
$$


Note that if $u \in C^{0}\left((0, T) ; L^{2}\left(\Omega, \mathbb{R}^{N}\right)\right)$ the slicewise means are defined for any $t \in(0, T)$. If $A$ is a ball $B_{\varrho}^{(\theta)}\left(x_{o}\right)$, we write $\langle u\rangle_{x_{o} ; \varrho}^{(\theta)}(t):=\langle u\rangle_{B_{\varrho}^{(\theta)}\left(x_{o}\right)}(t)$. Similarly, if $E$ is a cylinder of the form $Q_{\varrho}^{(\theta)}\left(z_{o}\right)$, we use the shorthand notation $(u)_{z_{o} ; \varrho}^{(\theta)}:=(u){ }_{Q_{\varrho}^{(\theta)}\left(z_{o}\right)}$.

2.2. General Setting and Results. We consider porous medium type systems of the form

$$
\partial_{t} u-\operatorname{div} \mathbf{A}\left(x, t, u, D \boldsymbol{u}^{m}\right)=\operatorname{div} F \quad \text { in } \Omega_{T},
$$

where $\mathbf{A}: \Omega_{T} \times \mathbb{R}^{N} \times \mathbb{R}^{N n} \rightarrow \mathbb{R}^{N n}$ is a Carathéodory vector field satisfying the following ellipticity and growth conditions that are modeled after the prototype system (1.2). For structural constants $0<\nu \leq L<\infty$, we assume that

$$
\left\{\begin{aligned}
\mathbf{A}(x, t, u, \xi) \cdot \xi & \geq \nu|\xi|^{2} \\
|\mathbf{A}(x, t, u, \xi)| & \leq L|\xi|
\end{aligned}\right.
$$

for a.e. $(x, t) \in \Omega_{T}$ and any $(u, \xi) \in \mathbb{R}^{N} \times \mathbb{R}^{N n}$. To formulate the main result, we introduce the notion of weak solution.

Definition 2.1. Let $m>0$ and $\mathbf{A}: \Omega_{T} \times \mathbb{R}^{N} \times \mathbb{R}^{N n} \rightarrow \mathbb{R}^{N n}$ be a vector field satisfying (2.3) and $F \in L^{2}\left(\Omega_{T}, \mathbb{R}^{N n}\right)$. A function

$$
u \in C^{0}\left((0, T) ; L^{1+m}\left(\Omega, \mathbb{R}^{N}\right)\right) \quad \text { with } \quad \boldsymbol{u}^{m} \in L^{2}\left(0, T ; W^{1,2}\left(\Omega, \mathbb{R}^{N}\right)\right)
$$

is a weak solution to the porous medium type system (2.2) if and only if the identity

$$
\iint_{\Omega_{T}}\left[u \cdot \partial_{t} \varphi-\mathbf{A}\left(x, t, u, D \boldsymbol{u}^{m}\right) \cdot D \varphi\right] \mathrm{d} x \mathrm{~d} t=\iint_{\Omega_{T}} F \cdot D \varphi \mathrm{d} x \mathrm{~d} t
$$

holds true, for any testing function $\varphi \in C_{0}^{\infty}\left(\Omega_{T}, \mathbb{R}^{N}\right)$.

Our main result reads as follows:

Theorem 2.2. Assume that

$$
m_{c}:=\frac{(n-2)_{+}}{n+2}<m \leq 1
$$

and $\sigma>2$. Then, there exists $\varepsilon_{o}=\varepsilon_{o}(n, m, \nu, L) \in(0,1]$ such that whenever $F \in$ $L^{\sigma}\left(\Omega_{T}, \mathbb{R}^{N n}\right)$ and $u$ is a weak solution of Equation (2.2) in the sense of Definition 2.1 under the assumptions (2.3), then with $\varepsilon_{1}:=\min \left\{\varepsilon_{o}, \sigma-2\right\}$ we have

$$
D \boldsymbol{u}^{m} \in L_{\text {loc }}^{2+\varepsilon_{1}}\left(\Omega_{T}, \mathbb{R}^{N n}\right) .
$$

Moreover, for every $\varepsilon \in\left(0, \varepsilon_{1}\right]$ and every cylinder $Q_{2 R}\left(z_{o}\right) \subseteq \Omega_{T}$, we have the quantitative local higher integrability estimate

$$
\begin{aligned}
\int_{Q_{R}}\left|D \boldsymbol{u}^{m}\right|^{2+\varepsilon} \mathrm{d} x \mathrm{~d} t \\
\leq c\left[1+\int_{Q_{2 R}}\left[\frac{|u|^{1+m}}{R^{\frac{1+m}{m}}}+|F|^{2}\right] \mathrm{d} x \mathrm{~d} t\right]^{\frac{\varepsilon d}{2}} \int_{Q_{2 R}}\left|D \boldsymbol{u}^{m}\right|^{2} \mathrm{~d} x \mathrm{~d} t \\
\quad+c \int_{Q_{2 R}}|F|^{2+\varepsilon} \mathrm{d} x \mathrm{~d} t .
\end{aligned}
$$

with $c=c(n, m, \nu, L) \geq 1$. Here,

$$
d:=\frac{2(1+m)}{2(1+m)-n(1-m)}
$$

denotes the scaling deficit. 
Remark 2.3. The scaling deficit $d$ that appears in the higher integrability estimate reflects the inhomogeneous scaling behavior of the porous medium system. In particular, we have $d=1$ if $m=1$, which corresponds to the case of the classical heat equation. On the other hand, in the case $n \geq 2$ we have $d \rightarrow \infty$ in the limit $m \downarrow m_{c}$. The latter underlines the significance of the lower bound $m>m_{c}$. If $m=1$, then (2.6) is similar to the reverse Hölder inequalities in [14] and [16] with $p=2$.

Remark 2.4. The above higher integrability result can easily be extended to the case of vector-fields A satisfying the more general growth and coercivity conditions

$$
\left\{\begin{array}{c}
\mathbf{A}(x, t, u, \xi) \cdot \xi \geq \nu|\xi|^{2}-h_{1}, \\
|\mathbf{A}(x, t, u, \xi)| \leq L|\xi|+h_{2},
\end{array}\right.
$$

with non-negative measurable functions $h_{1}, h_{2}: \Omega_{T} \rightarrow[0, \infty]$, so that $h_{1}+h_{2}^{2} \in$ $L^{\sigma / 2}\left(\Omega_{T}\right)$ for the exponent $\sigma>2$ from Theorem 2.2.

The quantitative local estimate (2.6) can easily be converted into an estimate on standard parabolic cylinders $C_{R}\left(z_{o}\right):=B_{R}\left(x_{o}\right) \times\left(t_{o}-R^{2}, t_{o}+R^{2}\right)$. The precise statement is:

Corollary 2.5. Under the assumptions of Theorem 2.2, on any cylinder $C_{2 R}\left(z_{o}\right) \subseteq \Omega_{T}$ and for every $\varepsilon \in\left(0, \varepsilon_{1}\right]$ we have

$$
\begin{aligned}
& R^{2+\varepsilon} \int_{C_{R}\left(z_{o}\right)}\left|D \boldsymbol{u}^{m}\right|^{2+\varepsilon} \mathrm{d} x \mathrm{~d} t \\
& \leq c R^{2}\left[1+\int_{C_{2 R}\left(z_{o}\right)}\left[|u|^{1+m}+R^{2}|F|^{2}\right] \mathrm{d} x \mathrm{~d} t\right]^{\frac{\varepsilon d}{2}} \iint_{C_{2 R}\left(z_{o}\right)}\left|D \boldsymbol{u}^{m}\right|^{2} \mathrm{~d} x \mathrm{~d} t \\
& \quad+c R^{2+\varepsilon} \oiiint_{C_{2 R}\left(z_{o}\right)}|F|^{2+\varepsilon} \mathrm{d} x \mathrm{~d} t
\end{aligned}
$$

for a constant $c=c(n, m, \nu, L)$.

\section{Auxiliary Material}

In this section we provide the necessary tools which will be used later. To "re-absorb" certain terms, we frequently shall use the following iteration lemma, cf. [15, Lemma 6.1].

Lemma 3.1. Let $0<\vartheta<1, A, C \geq 0$ and $\alpha>0$. Then there exists a constant $c=c(\alpha, \vartheta)$ such that for any non-negative bounded function $\phi:[r, \varrho] \rightarrow[0, \infty)$ with $0<r<\varrho$ satisfying

we have

$$
\phi(t) \leq \vartheta \phi(s)+\frac{A}{(s-t)^{\alpha}}+C \quad \text { for all } r \leq t<s \leq \varrho,
$$

$$
\phi(r) \leq c\left[\frac{A}{(\varrho-r)^{\alpha}}+C\right] .
$$

The following lemma can be deduced as in [15, Lemma 8.3].

Lemma 3.2. For any $\alpha>0$, there exists a constant $c=c(\alpha)$ such that, for all $a, b \in \mathbb{R}^{N}$, $N \in \mathbb{N}$, the following inequality holds true:

$$
\frac{1}{c}\left|\boldsymbol{b}^{\alpha}-\boldsymbol{a}^{\alpha}\right| \leq(|a|+|b|)^{\alpha-1}|b-a| \leq c\left|\boldsymbol{b}^{\alpha}-\boldsymbol{a}^{\alpha}\right| .
$$

The next lemma is an immediate consequence of Lemma 3.2.

Lemma 3.3. For any $\alpha \geq 1$, there exists a constant $c=c(\alpha)$ such that, for all $a, b \in \mathbb{R}^{N}$, $N \in \mathbb{N}$, the following inequality holds true:

$$
|b-a|^{\alpha} \leq c\left|\boldsymbol{b}^{\alpha}-\boldsymbol{a}^{\alpha}\right|
$$


It is well known that mean values over subsets $A \subset B$ are quasi-minimizers of the mapping $\mathbb{R}^{N} \ni a \mapsto \int_{B}|u-a|^{p} \mathrm{~d} x$. The following lemma shows that this also applies to powers $\boldsymbol{u}^{\alpha}$ of $u$, provided $\alpha \geq \frac{1}{p}$. For $p=2$ and $A=B$, the lemma has been proved in [9, Lemma 6.2]. The general version is established in [2, Lemma 3.5].

Lemma 3.4. For any $p \geq 1$ and $\alpha \geq \frac{1}{p}$, there exists a universal constant $c=c(\alpha, p)$ such that whenever $A \subset B \subset \mathbb{R}^{k}, k \in \mathbb{N}$, are two bounded domains with positive measure, then for any $u \in L^{\alpha p}\left(B, \mathbb{R}^{N}\right)$ and any $a \in \mathbb{R}^{N}$, we have

$$
f_{B}\left|\boldsymbol{u}^{\alpha}-(\boldsymbol{u})_{A}^{\alpha}\right|^{p} \mathrm{~d} x \leq \frac{c|B|}{|A|} f_{B}\left|\boldsymbol{u}^{\alpha}-\boldsymbol{a}^{\alpha}\right|^{p} \mathrm{~d} x .
$$

\section{ENERGY BOUNDS}

In this section we state an energy inequality and a gluing lemma. Both follow with standard arguments from the weak form (2.5) of the differential equation by testing with suitable testing functions. Later on, they will be used in the proof of Sobolev-Poincare and reverse Hölder type inequalities. At this point it should be emphasized, that these two lemmas are the only places in the proof of the higher integrability where the porous medium system is utilized. The proof of the energy estimate is along the lines of [3, Lemma 3.1], taking into account [3, Lemma 2.3 (i)] or [2, Lemma 3.4] and the different definition of scaled cylinders. The latter means that the radii $\varrho$ and $r$ in [3, Lemma 3.1] have to be replaced by $\theta^{\frac{m(m-1)}{1+m}} \varrho$ and $\theta^{\frac{m(m-1)}{1+m}} r$.

Lemma 4.1. Let $m>0$ and $u$ be a weak solution to (2.2) in $\Omega_{T}$ in the sense of Definition 2.1. Then, on any cylinder $Q_{\varrho}^{(\theta)}\left(z_{o}\right) \subseteq \Omega_{T}$ with $\varrho, \theta>0$, for any $r \in[\varrho / 2, \varrho)$ and any $a \in \mathbb{R}^{N}$, we have

$$
\begin{aligned}
& \sup _{t \in \Lambda_{r}\left(t_{o}\right)} f_{B_{r}^{(\theta)}\left(x_{o}\right)} \frac{\left|\boldsymbol{u}^{\frac{1+m}{2}}(t)-\boldsymbol{a}^{\frac{1+m}{2}}\right|^{2}}{r^{\frac{1+m}{m}}} \mathrm{~d} x+\int_{Q_{r}^{(\theta)}\left(z_{o}\right)}\left|D \boldsymbol{u}^{m}\right|^{2} \mathrm{~d} x \mathrm{~d} t \\
& \leq c \int_{Q_{\varrho}^{(\theta)}\left(z_{o}\right)}\left[\frac{\left|\boldsymbol{u}^{\frac{1+m}{2}}-\boldsymbol{a}^{\frac{1+m}{2}}\right|^{2}}{\varrho^{\frac{1+m}{m}}-r^{\frac{1+m}{m}}}+\frac{\left|\boldsymbol{u}^{m}-\boldsymbol{a}^{m}\right|^{2}}{\theta^{\frac{2 m(m-1)}{1+m}}(\varrho-r)^{2}}+|F|^{2}\right] \mathrm{d} x \mathrm{~d} t,
\end{aligned}
$$

where $c=c(m, \nu, L)$.

The following lemma serves to compare the slice-wise mean values of a given weak solution at different times. It is often called gluing lemma. Such an assertion is necessary and very useful since Poincaré's and Sobolev's inequality can only be applied slice-wise. The proof is exactly as in [3, Lemma 3.2], taking into account the different definition of scaled cylinders.

Lemma 4.2. Let $m>0$ and $u$ be a weak solution to (2.2) in $\Omega_{T}$ in the sense of Definition 2.1. Then, for any cylinder $Q_{\varrho}^{(\theta)}\left(z_{o}\right) \subseteq \Omega_{T}$ with $\varrho, \theta>0$ there exists $\hat{\varrho} \in\left[\frac{\varrho}{2}, \varrho\right]$ such that for all $t_{1}, t_{2} \in \Lambda_{\varrho}\left(t_{o}\right)$ we have

$$
\left|\langle u\rangle_{x_{o} ; \hat{\varrho}}^{(\theta)}\left(t_{2}\right)-\langle u\rangle_{x_{o} ; \hat{\varrho}}^{(\theta)}\left(t_{1}\right)\right| \leq c \theta^{\frac{m(1-m)}{1+m}} \varrho^{\frac{1}{m}} \iint_{Q_{\varrho}^{(\theta)}\left(z_{o}\right)}\left[\left|D \boldsymbol{u}^{m}\right|+|F|\right] \mathrm{d} x \mathrm{~d} t,
$$

for a constant $c=c(L)$.

\section{SoboleV-Poincaré TYPE INEQUALITY}

In this section we consider cylinders $Q_{\varrho}^{(\theta)}\left(z_{o}\right) \subseteq \Omega_{T}$, where $\varrho, \theta>0$, which satisfy a sub-intrinsic coupling in the sense that for some constant $K \geq 1$ we have

$$
\iint_{Q_{\varrho}^{(\theta)}\left(z_{o}\right)} \frac{|u|^{1+m}}{\varrho^{\frac{1+m}{m}}} \mathrm{~d} x \mathrm{~d} t \leq K \theta^{2 m} .
$$


Furthermore, we assume that either

(5.2) $\theta^{2 m} \leq K \int_{Q_{\varrho}^{(\theta)}\left(z_{o}\right)} \frac{|u|^{1+m}}{\varrho^{\frac{1+m}{m}}} \mathrm{~d} x \mathrm{~d} t \quad$ or $\quad \theta^{2 m} \leq K \int_{Q_{\varrho}^{(\theta)}\left(z_{o}\right)}\left[\left|D \boldsymbol{u}^{m}\right|^{2}+|F|^{2}\right] \mathrm{d} x \mathrm{~d} t$

holds true. The principal goal of the section is to establish the following Sobolev-Poincaré type inequality. This inequality illustrates the significance of the lower bound $m>m_{c}$, since only in this case, we obtain an integrability exponent $2 q<2$ on the right-hand side.

Lemma 5.1. Let $m \in\left(m_{c}, 1\right]$ and $u$ be a weak solution to (2.2) in $\Omega_{T}$ in the sense of Definition 2.1. Then, on any cylinder $Q_{\varrho}^{(\theta)}\left(z_{o}\right) \subseteq \Omega_{T}$ satisfying (5.1), with $\varrho, \theta>0$, and for any $\varepsilon \in(0,1]$, we have

$$
\begin{aligned}
& \int_{Q_{\varrho}^{(\theta)}\left(z_{o}\right)} \frac{\left|\boldsymbol{u}^{\frac{1+m}{2}}-\left(\boldsymbol{u}^{\frac{1+m}{2}}\right)_{z_{o} ; \varrho}^{(\theta)}\right|^{2}}{\varrho^{\frac{1+m}{m}}} \mathrm{~d} x \mathrm{~d} t \\
& \leq \varepsilon\left[\sup _{t \in \Lambda_{\varrho}\left(t_{o}\right)} f_{B_{\varrho}^{(\theta)}\left(x_{o}\right)} \frac{\left|\boldsymbol{u}^{\frac{1+m}{2}}(t)-\left(\boldsymbol{u}^{\frac{1+m}{2}}\right)_{z_{o} ; \varrho}^{(\theta)}\right|^{2}}{\varrho^{\frac{1+m}{m}}} \mathrm{~d} x+\int_{Q_{\varrho}^{(\theta)}\left(z_{o}\right)}\left|D \boldsymbol{u}^{m}\right|^{2} \mathrm{~d} x \mathrm{~d} t\right] \\
& \quad+\frac{c}{\varepsilon^{\frac{2}{n}}}\left[\left[\int_{Q_{\varrho}^{(\theta)}\left(z_{o}\right)}\left|D \boldsymbol{u}^{m}\right|^{2 q} \mathrm{~d} x \mathrm{~d} t\right]^{\frac{1}{q}}+\iint_{Q_{\varrho}^{(\theta)}\left(z_{o}\right)}|F|^{2} \mathrm{~d} x \mathrm{~d} t\right]
\end{aligned}
$$

for a constant $c=c(n, m, L, K)$. Here the integrability exponent $q$ is given by

$$
q:=\max \left\{\frac{n(1+m)}{2(n m+1+m)}, \frac{1}{2}\right\}<1 .
$$

Proof. Throughout the proof we omit the center $z_{o}$ in our notation. By $\hat{\varrho} \in\left[\frac{1}{2} \varrho, \varrho\right]$ we denote the radius introduced in Lemma 4.2. We start our considerations by estimating

$$
\iint_{Q_{\varrho}^{(\theta)}} \frac{\left|\boldsymbol{u}^{\frac{1+m}{2}}-\left(\boldsymbol{u}^{\frac{1+m}{2}}\right)_{\varrho}^{(\theta)}\right|^{2}}{\varrho^{\frac{1+m}{m}}} \mathrm{~d} x \mathrm{~d} t \leq \iint_{Q_{\varrho}^{(\theta)}} \frac{\left|\boldsymbol{u}^{\frac{1+m}{2}}-\left[(\boldsymbol{u})_{\hat{\varrho}}^{(\theta)}\right]^{\frac{1+m}{2}}\right|^{2}}{\varrho^{\frac{1+m}{m}}} \mathrm{~d} x \mathrm{~d} t \leq 2[\mathrm{I}+\mathrm{II}] .
$$

Here we have abbreviated

$$
\begin{gathered}
\mathrm{I}:=\int_{Q_{\varrho}^{(\theta)}} \frac{\left|\boldsymbol{u}^{\frac{1+m}{2}}-\left[\langle\boldsymbol{u}\rangle_{\hat{\varrho}}^{(\boldsymbol{\theta})}(\boldsymbol{t})\right]^{\frac{1+m}{2}}\right|^{2}}{\varrho^{\frac{1+m}{m}}} \mathrm{~d} x \mathrm{~d} t, \\
\mathrm{II}:=f_{\Lambda_{\varrho}} \frac{\left|\left[\langle\boldsymbol{u}\rangle_{\hat{\varrho}}^{(\boldsymbol{\theta})}(\boldsymbol{t})\right]^{\frac{1+m}{2}}-\left[(\boldsymbol{u})_{\hat{\varrho}}^{(\boldsymbol{\theta})}\right]^{\frac{1+m}{2}}\right|^{2}}{\varrho^{\frac{1+m}{m}}} \mathrm{~d} t .
\end{gathered}
$$

The first term can be estimated with Young's inequality and Lemma 3.4. We obtain

$$
\begin{aligned}
\mathrm{I} \leq & \frac{1}{\varrho^{\frac{1+m}{m}}} \sup _{t \in \Lambda_{\varrho}}\left[f_{B_{\varrho}^{(\theta)}}\left|\boldsymbol{u}^{\frac{1+m}{2}}-\left[\langle\boldsymbol{u}\rangle_{\hat{\varrho}}^{(\boldsymbol{\theta})}(\boldsymbol{t})\right]^{\frac{1+m}{2}}\right|^{2} \mathrm{~d} x\right]^{\frac{2}{n+2}} \\
& \cdot f_{\Lambda_{\varrho}}\left[f_{B_{\varrho}^{(\theta)}}\left|\boldsymbol{u}^{\frac{1+m}{2}}-\left[\langle\boldsymbol{u}\rangle_{\hat{\varrho}}^{(\boldsymbol{\theta})}(\boldsymbol{t})\right]^{\frac{1+m}{2}}\right|^{2} \mathrm{~d} x\right]^{\frac{n}{n+2}} \mathrm{~d} t \\
\leq & \varepsilon \sup _{t \in \Lambda_{\varrho}} f_{B_{\varrho}^{(\theta)}} \frac{\left|\boldsymbol{u}^{\frac{1+m}{2}}(t)-\left(\boldsymbol{u}^{\frac{1+m}{2}}\right)_{\varrho}^{(\theta)}\right|^{2}}{\varrho^{\frac{1+m}{m}}} \mathrm{~d} x+\frac{c}{\varepsilon^{\frac{2}{n}} \varrho^{\frac{1+m}{m}}} \mathrm{III}^{\frac{n+2}{n}},
\end{aligned}
$$

where $c=c(n, m)$ and

$$
\text { III }:=f_{\Lambda_{\varrho}}\left[f_{B_{\varrho}^{(\theta)}}\left|\boldsymbol{u}^{\frac{1+m}{2}}-\left[\left\langle\boldsymbol{u}^{\boldsymbol{m}}\right\rangle_{\varrho}^{(\boldsymbol{\theta})}(\boldsymbol{t})\right]^{\frac{1+m}{2 m}}\right|^{2} \mathrm{~d} x\right]^{\frac{n}{n+2}} \mathrm{~d} t
$$


If $m<1$ we estimate the integral III by means of Lemma 3.2 with $\alpha=\frac{1+m}{2 m}$ and Hölder's inequality in space with exponents $\frac{1+m}{1-m}$ and $\frac{1+m}{2 m}$, which yields

$$
\begin{aligned}
\mathrm{III} & \leq f_{\Lambda_{\varrho}}\left[f_{B_{\varrho}^{(\theta)}}\left(\left|\boldsymbol{u}^{m}\right|+\left|\left\langle\boldsymbol{u}^{m}\right\rangle_{\varrho}^{(\theta)}(t)\right|\right)^{\frac{1-m}{m}}\left|\boldsymbol{u}^{m}-\left\langle\boldsymbol{u}^{m}\right\rangle_{\varrho}^{(\theta)}(t)\right|^{2} \mathrm{~d} x\right]^{\frac{n}{n+2}} \mathrm{~d} t \\
& \leq f_{\Lambda_{\varrho}}\left[f_{B_{\varrho}^{(\theta)}}|u|^{1+m} \mathrm{~d} x\right]^{\frac{n}{n+2} \frac{1-m}{1+m}}\left[f_{B_{\varrho}^{(\theta)}}\left|\boldsymbol{u}^{m}-\left\langle\boldsymbol{u}^{m}\right\rangle_{\varrho}^{(\theta)}(t)\right|^{\frac{1+m}{m}} \mathrm{~d} x\right]^{\frac{n}{n+2} \frac{2 m}{1+m}} \mathrm{~d} t .
\end{aligned}
$$

To proceed further, we recall the definition of $q$. Now, again in the case $m<1$, we apply Hölder's inequality in time with exponents $\frac{(n+2)(1+m)}{n(1-m)}$ and $\frac{(n+2)(1+m)}{2(n m+1+m)} \leq \frac{q(n+2)}{n}$, the sub-intrinsic coupling (5.1) and Sobolev's inequality on the time slices. This leads to

$$
\begin{aligned}
\mathrm{III} & \leq\left[\int_{Q_{\varrho}^{(\theta)}}|u|^{1+m} \mathrm{~d} x \mathrm{~d} t\right]^{\frac{n(1-m)}{(n+2)(1+m)}} \\
& \cdot\left[f_{\Lambda_{\varrho}}\left[f_{B_{\varrho}^{(\theta)}}\left|\boldsymbol{u}^{m}-\left\langle\boldsymbol{u}^{m}\right\rangle_{\varrho}^{(\theta)}(t)\right|^{\frac{1+m}{m}} \mathrm{~d} x\right]^{\frac{2 m q}{1+m}} \mathrm{~d} t\right]^{\frac{n}{q(n+2)}} \\
& \leq c\left(\theta^{2 m} \varrho^{\frac{1+m}{m}}\right)^{\frac{n(1-m)}{(n+2)(1+m)}}\left(\theta^{\frac{m(m-1)}{1+m}} \varrho\right)^{\frac{2 n}{n+2}}\left[\int_{Q_{\varrho}^{(\theta)}}\left|D \boldsymbol{u}^{m}\right|^{2 q} \mathrm{~d} x \mathrm{~d} t\right]^{\frac{n}{q(n+2)}} \\
& =c\left[\varrho^{\frac{1+m}{m}}\left[\int_{Q_{\varrho}^{(\theta)}}\left|D \boldsymbol{u}^{m}\right|^{2 q} \mathrm{~d} x \mathrm{~d} t\right]^{\frac{1}{q}}\right]^{\frac{n}{n+2}}
\end{aligned}
$$

where $c=c(n, m, K)$. Note that this inequality also holds true for $m=1$. In this case we directly apply Sobolev's inequality on the time slices. In any case, the combination of the last inequality with (5.4) yields

$$
\mathrm{I} \leq \varepsilon \sup _{t \in \Lambda_{\varrho}} f_{B_{\varrho}^{(\theta)}} \frac{\left|\boldsymbol{u}^{\frac{1+m}{2}}(t)-\left(\boldsymbol{u}^{\frac{1+m}{2}}\right)_{\varrho}^{(\theta)}\right|^{2}}{\varrho^{\frac{1+m}{m}}} \mathrm{~d} x+\frac{c}{\varepsilon^{\frac{2}{n}}}\left[\int_{Q_{\varrho}^{(\theta)}}\left|D \boldsymbol{u}^{m}\right|^{2 q} \mathrm{~d} x \mathrm{~d} t\right]^{\frac{1}{q}} .
$$

It remains to estimate II. To this end, we use the fact $\frac{1+m}{2} \leq 1$ in Lemma 3.3 and the gluing Lemma 4.2 to deduce

$$
\begin{aligned}
\mathrm{II} & \leq c f_{\Lambda_{\varrho}} \frac{\left|\langle u\rangle_{\hat{\varrho}}^{(\theta)}(t)-(u)_{\hat{\varrho}}^{(\theta)}\right|^{1+m}}{\varrho^{\frac{1+m}{m}}} \mathrm{~d} t \\
& \leq \frac{c}{\varrho^{\frac{1+m}{m}}} \int_{\Lambda_{\varrho}} f_{\Lambda_{\varrho}}\left|\langle u\rangle_{\hat{\varrho}}^{(\theta)}(t)-\langle u\rangle_{\hat{\varrho}}^{(\theta)}(\tau)\right|^{1+m} \mathrm{~d} t \mathrm{~d} \tau \\
& \leq c \theta^{m(1-m)}\left[\int_{Q_{\varrho}^{(\theta)}}\left[\left|D \boldsymbol{u}^{m}\right|+|F|\right] \mathrm{d} x \mathrm{~d} t\right]^{1+m}
\end{aligned}
$$

for a constant $c=c(m, L)$. If either (5.2) 2 is satisfied or if $m=1$, then we have

$$
\begin{aligned}
\mathrm{II} & \leq c\left[\int_{Q_{\varrho}^{(\theta)}}\left[\left|D \boldsymbol{u}^{m}\right|^{2}+|F|^{2}\right] \mathrm{d} x \mathrm{~d} t\right]^{\frac{1-m}{2}}\left[\iint_{Q_{\varrho}^{(\theta)}}\left[\left|D \boldsymbol{u}^{m}\right|+|F|\right] \mathrm{d} x \mathrm{~d} t\right]^{1+m} \\
& \leq \varepsilon \iint_{Q_{\varrho}^{(\theta)}}\left|D \boldsymbol{u}^{m}\right|^{2} \mathrm{~d} x \mathrm{~d} t+\frac{c}{\varepsilon^{\frac{1-m}{1+m}}}\left[\left[\int_{Q_{\varrho}^{(\theta)}}\left|D \boldsymbol{u}^{m}\right|^{2 q} \mathrm{~d} x \mathrm{~d} t\right]^{\frac{1}{q}}+\int_{Q_{\varrho}^{(\theta)}}|F|^{2} \mathrm{~d} x \mathrm{~d} t\right],
\end{aligned}
$$

where the constant $c$ depends only on $m, L$ and $K$. Together with the estimate for I, this proves the asserted inequality. Note that $\frac{1-m}{1+m}<\frac{2}{n}$ since $m>m_{c}$. Otherwise, if $m<1$ and $(5.2)_{1}$ is satisfied, then we argue as follows. First, observe that

$$
\theta^{2 m} \leq 2 K \int_{Q_{\varrho}^{(\theta)}} \frac{\left|\boldsymbol{u}^{\frac{1+m}{2}}-\left[(\boldsymbol{u})_{\hat{\varrho}}^{(\boldsymbol{\theta})}\right]^{\frac{1+m}{2}}\right|^{2}}{\varrho^{\frac{1+m}{m}}} \mathrm{~d} x \mathrm{~d} t+\frac{2 K\left|(u)_{\hat{\varrho}}^{(\theta)}\right|^{1+m}}{\varrho^{\frac{1+m}{m}}} .
$$


Therefore, we have

$$
\mathrm{II}=\frac{\theta^{\frac{2 m(1-m)}{1+m}} \mathrm{II}}{\theta^{\frac{2 m(1-m)}{1+m}}} \leq c\left[\mathrm{II}_{1}+\mathrm{II}_{2}\right]
$$

with

$$
\begin{aligned}
& \mathrm{II}_{1}:=\frac{1}{\theta^{\frac{2 m(1-m)}{1+m}}}\left[\iint_{Q_{\varrho}^{(\theta)}} \frac{\left|\boldsymbol{u}^{\frac{1+m}{2}}-\left[(\boldsymbol{u})_{\hat{\varrho}}^{(\boldsymbol{\theta})}\right]^{\frac{1+m}{2}}\right|^{2}}{\varrho^{\frac{1+m}{m}}} \mathrm{~d} x \mathrm{~d} t\right]^{\frac{1-m}{1+m}} \cdot \mathrm{II}, \\
& \mathrm{II}_{2}:=\frac{\left|(u)_{\hat{\varrho}}^{(\theta)}\right|^{1-m}}{\theta^{\frac{2 m(1-m)}{1+m}} \varrho^{\frac{1-m}{m}}} \cdot \text { II. }
\end{aligned}
$$

To estimate $\mathrm{II}_{1}$, we apply in turn (5.5), assumption (5.1), Lemma 3.4 and Young's inequality with exponents $\frac{2}{1-m}, \frac{2}{1+m}$. This gives

$$
\begin{aligned}
\mathrm{II}_{1} & \leq \frac{c}{\theta^{\frac{m(1-m)^{2}}{1+m}}}\left[\iint_{Q_{\varrho}^{(\theta)}} \frac{\left|\boldsymbol{u}^{\frac{1+m}{2}}-\left[(\boldsymbol{u})_{\hat{\varrho}}^{(\boldsymbol{\theta})}\right]^{\frac{1+m}{2}}\right|^{2}}{\varrho^{\frac{1+m}{m}}} \mathrm{~d} x \mathrm{~d} t\right]^{\frac{1-m}{1+m}} \\
\cdot\left[\iint_{Q_{\varrho}^{(\theta)}}\left[\left|D \boldsymbol{u}^{m}\right|+|F|\right] \mathrm{d} x \mathrm{~d} t\right]^{1+m} & \\
& \leq c\left[\int_{Q_{\varrho}^{(\theta)}} \frac{\left|\boldsymbol{u}^{\frac{1+m}{2}}-\left[(\boldsymbol{u})_{\hat{\varrho}}^{(\theta)}\right]^{\frac{1+m}{2}}\right|^{2}}{\varrho^{\frac{1+m}{m}}} \mathrm{~d} x \mathrm{~d} t\right]^{\frac{1-m}{2}}\left[\iint_{Q_{\varrho}^{(\theta)}}\left[\left|D \boldsymbol{u}^{m}\right|+|F|\right] \mathrm{d} x \mathrm{~d} t\right]^{1+m} \\
& \leq \frac{1}{2} \int_{Q_{\varrho}^{(\theta)}} \frac{\left|\boldsymbol{u}^{\frac{1+m}{2}}-\left(\boldsymbol{u}^{\frac{1+m}{2}}\right)_{\varrho}^{(\theta)}\right|^{2}}{\varrho^{\frac{1+m}{m}}} \mathrm{~d} x \mathrm{~d} t+c\left[\int_{Q_{\varrho}^{(\theta)}}\left[\left|D \boldsymbol{u}^{m}\right|+|F|\right] \mathrm{d} x \mathrm{~d} t\right]^{2},
\end{aligned}
$$

with a constant $c=c(m, L, K)$. For the term $\mathrm{II}_{2}$, we proceed as follows. We first insert the expression for the term II, then use Lemma 3.2 with $\alpha:=\frac{2}{1+m}$, and finally apply the gluing Lemma 4.2. This leads to

$$
\begin{aligned}
\mathrm{II}_{2} & \leq \frac{c}{\theta^{\frac{2 m(1-m)}{1+m}} \varrho^{\frac{2}{m}}} f_{\Lambda_{\varrho}}\left|(u)_{\hat{\varrho}}^{(\theta)}\right|^{1-m}\left|\left[\langle\boldsymbol{u}\rangle_{\hat{\varrho}}^{(\boldsymbol{\theta})}(\boldsymbol{t})\right]^{\frac{1+m}{2}}-\left[(\boldsymbol{u})_{\hat{\varrho}}^{(\boldsymbol{\theta})}\right]^{\frac{1+m}{2}}\right|^{2} \mathrm{~d} t \\
& \leq \frac{c}{\theta^{\frac{2 m(1-m)}{1+m}} \varrho^{\frac{2}{m}}} f_{\Lambda_{\varrho}}\left|\langle u\rangle_{\hat{\varrho}}^{(\theta)}(t)-(u)_{\hat{\varrho}}^{(\theta)}\right|^{2} \mathrm{~d} t \\
& \leq \frac{c}{\theta^{\frac{2 m(1-m)}{1+m}} \varrho^{\frac{2}{m}}} \int_{\Lambda_{\varrho}} f_{\Lambda_{\varrho}}\left|\langle u\rangle_{\hat{\varrho}}^{(\theta)}(t)-\langle u\rangle_{\hat{\varrho}}^{(\theta)}(\tau)\right|^{2} \mathrm{~d} t \mathrm{~d} \tau \\
& \leq c\left[\int_{Q_{\varrho}^{(\theta)}}\left[\left|D \boldsymbol{u}^{m}\right|+|F|\right] \mathrm{d} x \mathrm{~d} t\right]^{2},
\end{aligned}
$$

again with a constant $c=c(m, L, K)$. Collecting the estimates for $\mathrm{I}_{1} \mathrm{II}_{1}$, and $\mathrm{II}_{2}$, we arrive at

$$
\begin{aligned}
\iint_{Q_{\varrho}^{(\theta)}} & \frac{\left|\boldsymbol{u}^{\frac{1+m}{2}}-\left(\boldsymbol{u}^{\frac{1+m}{2}}\right)_{\varrho}^{(\theta)}\right|^{2}}{\varrho^{\frac{1+m}{m}}} \mathrm{~d} x \mathrm{~d} t \\
\leq & \varepsilon \sup _{t \in \Lambda_{\varrho}} \int_{B_{\varrho}^{(\theta)}} \frac{\left|\boldsymbol{u}^{\frac{1+m}{2}}(t)-\left(\boldsymbol{u}^{\frac{1+m}{2}}\right)_{\varrho}^{(\theta)}\right|^{2}}{\varrho^{\frac{1+m}{m}}} \mathrm{~d} x+\frac{c}{\varepsilon^{\frac{2}{n}}}\left[\iint_{Q_{\varrho}^{(\theta)}}\left|D \boldsymbol{u}^{m}\right|^{2 q} \mathrm{~d} x \mathrm{~d} t\right]^{\frac{1}{q}} \\
& +\frac{1}{2} \int_{Q_{\varrho}^{(\theta)}} \frac{\left|\boldsymbol{u}^{\frac{1+m}{2}}-\left(\boldsymbol{u}^{\frac{1+m}{2}}\right)_{\varrho}^{(\theta)}\right|^{2}}{\varrho^{\frac{1+m}{m}}} \mathrm{~d} x \mathrm{~d} t+c\left[\iint_{Q_{\varrho}^{(\theta)}}\left[\left|D \boldsymbol{u}^{m}\right|+|F|\right] \mathrm{d} x \mathrm{~d} t\right]^{2} .
\end{aligned}
$$

Re-absorbing the second last term into the left-hand side, and applying in turn Hölder's inequality we again obtain the asserted Sobolev-Poincaré inequality. 


\section{REVERSE HÖLDER INEQUALITY}

The core of any proof of higher integrability of the gradient is a reverse Hölder inequality. In this section we establish such an inequality on certain intrinsic cylinders. Throughout this section we assume that $Q_{2 \varrho}^{(\theta)}\left(z_{o}\right) \subseteq \Omega_{T}$ with $\varrho, \theta>0$ is a scaled cylinder satisfying a sub-intrinsic coupling

$$
\int_{Q_{2 \varrho}^{(\theta)}\left(z_{o}\right)} \frac{|u|^{1+m}}{(2 \varrho)^{\frac{1+m}{m}}} \mathrm{~d} x \mathrm{~d} t \leq K \theta^{2 m}
$$

for some constant $K \geq 1$. Furthermore, we assume that either

(6.2) $\theta^{2 m} \leq K \int_{Q_{\varrho}^{(\theta)}\left(z_{o}\right)} \frac{|u|^{1+m}}{\varrho^{\frac{1+m}{m}}} \mathrm{~d} x \mathrm{~d} t \quad$ or $\quad \theta^{2 m} \leq K \int_{Q_{\varrho}^{(\theta)}\left(z_{o}\right)}\left[\left|D \boldsymbol{u}^{m}\right|^{2}+|F|^{2}\right] \mathrm{d} x \mathrm{~d} t$.

This specifies the setup for the following reverse Hölder inequality.

Proposition 6.1. Let $m \in\left(m_{c}, 1\right]$ and $u$ be a weak solution to (2.2) in $\Omega_{T}$ in the sense of Definition 2.1. Then, on any cylinder $Q_{2 \varrho}^{(\theta)}\left(z_{o}\right) \subseteq \Omega_{T}$ with $\varrho, \theta>0$ satisfying (6.1) and (6.2), we have

$$
\int_{Q_{\varrho}^{(\theta)}\left(z_{o}\right)}\left|D \boldsymbol{u}^{m}\right|^{2} \mathrm{~d} x \mathrm{~d} t \leq c\left[\iint_{Q_{2 \varrho}^{(\theta)}\left(z_{o}\right)}\left|D \boldsymbol{u}^{m}\right|^{2 q} \mathrm{~d} x \mathrm{~d} t\right]^{\frac{1}{q}}+c \int_{Q_{2 \varrho}^{(\theta)}\left(z_{o}\right)}|F|^{2} \mathrm{~d} x \mathrm{~d} t,
$$

for a constant $c=c(n, m, \nu, L, K)$. Here, $q<1$ is the integrability exponent from (5.3).

Proof. We omit the reference to the center $z_{o}$ in the notation and consider radii $r, s$ with $\varrho \leq r<s \leq 2 \varrho$. Note that hypothesis (6.1) and (6.2) imply that the coupling conditions (5.1) and (5.2) are satisfied on $Q_{s}^{(\theta)}$ with constant $2^{n+2+\frac{2}{m}} K$ instead of $K$. From the energy estimate in Lemma 4.1, we obtain with a constant $c=c(m, \nu, L)$ that

$$
\begin{aligned}
& \sup _{t \in \Lambda_{r}} \int_{B_{r}^{(\theta)}} \frac{\left|\boldsymbol{u}^{\frac{1+m}{2}}(t)-\left(\boldsymbol{u}^{\frac{1+m}{2}}\right)_{r}^{(\theta)}\right|^{2}}{r^{\frac{1+m}{m}}} \mathrm{~d} x+\iint_{Q_{r}^{(\theta)}}\left|D \boldsymbol{u}^{m}\right|^{2} \mathrm{~d} x \mathrm{~d} t \\
& \leq c \int_{Q_{s}^{(\theta)}} \frac{\left|\boldsymbol{u}^{\frac{1+m}{2}}-\left(\boldsymbol{u}^{\frac{1+m}{2}}\right)_{r}^{(\theta)}\right|^{2}}{s^{\frac{1+m}{m}}-r^{\frac{1+m}{m}}} \mathrm{~d} x \mathrm{~d} t+c \iint_{Q_{s}^{(\theta)}} \frac{\left|\boldsymbol{u}^{m}-\left[\left(\boldsymbol{u}^{\frac{1+m}{2}}\right)_{r}^{(\theta)}\right]^{\frac{2 m}{1+m}}\right|^{2}}{\theta^{\frac{2 m(m-1)}{1+m}}(s-r)^{2}} \mathrm{~d} x \mathrm{~d} t \\
& \quad+c \int_{Q_{s}^{(\theta)}}|F|^{2} \mathrm{~d} x \mathrm{~d} t \\
& =: \mathrm{I}+\mathrm{II}+\mathrm{III},
\end{aligned}
$$

where the meaning of I, II and III is clear in this context. We let

$$
\mathcal{R}_{r, s}:=\frac{s}{s-r} .
$$

To estimate the term I we first observe that $(s-r)^{\frac{1+m}{m}} \leq s^{\frac{1+m}{m}}-r^{\frac{1+m}{m}}$. This, together with an application of Lemma 3.4 implies

$$
\mathrm{I} \leq c \mathcal{R}_{r, s}^{\frac{1+m}{m}} \iint_{Q_{s}^{(\theta)}} \frac{\left|\boldsymbol{u}^{\frac{1+m}{2}}-\left(\boldsymbol{u}^{\frac{1+m}{2}}\right)_{s}^{(\theta)}\right|^{2}}{s^{\frac{1+m}{m}}} \mathrm{~d} x \mathrm{~d} t
$$

again with a constant $c$ depending on $m, \nu, L$ only. We now turn our attention to the term II, which we re-write as

$$
\mathrm{II}=c \mathcal{R}_{r, s}^{2} \theta^{\frac{2 m(1-m)}{1+m}} \int_{Q_{s}^{(\theta)}} \frac{\left|\boldsymbol{u}^{m}-\left[\left(\boldsymbol{u}^{\frac{1+m}{2}}\right)_{r}^{(\boldsymbol{\theta})}\right]^{\frac{2 m}{1+m}}\right|^{2}}{s^{2}} \mathrm{~d} x \mathrm{~d} t .
$$


If (6.2) 2 is satisfied, we apply Lemma 3.3, Hölder's inequality, Lemma 3.4 and Young's inequality to obtain for $\varepsilon \in(0,1]$ that

$$
\begin{aligned}
\mathrm{II} & \leq c \mathcal{R}_{r, s}^{2} \theta^{\frac{2 m(1-m)}{1+m}}\left[\iint_{Q_{s}^{(\theta)}} \frac{\left|\boldsymbol{u}^{\frac{1+m}{2}}-\left(\boldsymbol{u}^{\frac{1+m}{2}}\right)_{r}^{(\theta)}\right|^{2}}{s^{\frac{1+m}{m}}} \mathrm{~d} x \mathrm{~d} t\right]^{\frac{2 m}{1+m}} \\
& \leq c \mathcal{R}_{r, s}^{2}\left[\iint_{Q_{\varrho}^{(\theta)}}\left[\left|D \boldsymbol{u}^{m}\right|^{2}+|F|^{2}\right] \mathrm{d} x \mathrm{~d} t\right]^{\frac{1-m}{1+m}}\left[\iint_{Q_{s}^{(\theta)}} \frac{\left|\boldsymbol{u}^{\frac{1+m}{2}}-\left(\boldsymbol{u}^{\frac{1+m}{2}}\right)_{s}^{(\theta)}\right|^{2}}{s^{\frac{1+m}{m}}} \mathrm{~d} x \mathrm{~d} t\right]^{\frac{2 m}{1+m}} \\
& \leq \varepsilon \mathcal{R}_{r, s}^{2} \int_{Q_{s}^{(\theta)}}\left[\left|D \boldsymbol{u}^{m}\right|^{2}+|F|^{2}\right] \mathrm{d} x \mathrm{~d} t+\frac{c \mathcal{R}_{r, s}^{2}}{\varepsilon^{\frac{1-m}{2 m}}} \iint_{Q_{s}^{(\theta)}} \frac{\left|\boldsymbol{u}^{\frac{1+m}{2}}-\left(\boldsymbol{u}^{\frac{1+m}{2}}\right)_{s}^{(\theta)}\right|^{2}}{s^{\frac{1+m}{m}}} \mathrm{~d} x \mathrm{~d} t
\end{aligned}
$$

with $c=c(m, \nu, L, K)$. In the case $m=1$, this estimate follows even without an application of Young's inequality. Otherwise, if $(6.2)_{1}$ is in force, we have that

$$
\begin{aligned}
\theta^{2 m} & \leq 2^{n+2+\frac{2}{m}} K \int_{Q_{s}^{(\theta)}} \frac{|u|^{1+m}}{s^{\frac{1+m}{m}}} \mathrm{~d} x \mathrm{~d} t \\
& \leq c \int_{Q_{s}^{(\theta)}} \frac{\left|\boldsymbol{u}^{\frac{1+m}{2}}-\left(\boldsymbol{u}^{\frac{1+m}{2}}\right)_{r}^{(\theta)}\right|^{2}}{s^{\frac{1+m}{m}}} \mathrm{~d} x \mathrm{~d} t+\frac{c\left|\left(\boldsymbol{u}^{\frac{1+m}{2}}\right)_{r}^{(\theta)}\right|^{2}}{s^{\frac{1+m}{m}}} .
\end{aligned}
$$

This leads to

$$
\mathrm{II} \leq c \mathcal{R}_{r, s}^{2}\left[\mathrm{II}_{1}+\mathrm{II}_{2}\right]
$$

where we have set

$$
\mathrm{II}_{1}:=\left[\iint_{Q_{s}^{(\theta)}} \frac{\left|\boldsymbol{u}^{\frac{1+m}{2}}-\left(\boldsymbol{u}^{\frac{1+m}{2}}\right)_{r}^{(\theta)}\right|^{2}}{s^{\frac{1+m}{m}}} \mathrm{~d} x \mathrm{~d} t\right]^{\frac{1-m}{1+m}} \iint_{Q_{s}^{(\theta)}} \frac{\left|\boldsymbol{u}^{m}-\left[\left(\boldsymbol{u}^{\frac{1+m}{2}}\right)_{r}^{(\boldsymbol{\theta})}\right]^{\frac{2 m}{1+m}}\right|^{2}}{s^{2}} \mathrm{~d} x \mathrm{~d} t
$$

and

$$
\mathrm{II}_{2}:=\frac{\left|\left(\boldsymbol{u}^{\frac{1+m}{2}}\right)_{r}^{(\theta)}\right|^{\frac{2(1-m)}{1+m}}}{s^{\frac{1-m}{m}}} \iint_{Q_{s}^{(\theta)}} \frac{\left|\boldsymbol{u}^{m}-\left[\left(\boldsymbol{u}^{\frac{1+m}{2}}\right)_{r}^{(\boldsymbol{\theta})}\right]^{\frac{2 m}{1+m}}\right|^{2}}{s^{2}} \mathrm{~d} x \mathrm{~d} t .
$$

To term $\mathrm{II}_{1}$ we apply in turn Lemma 3.3, Hölder's inequality and Lemma 3.4, and obtain

$$
\mathrm{II}_{1} \leq c \int_{Q_{s}^{(\theta)}} \frac{\left|\boldsymbol{u}^{\frac{1+m}{2}}-\left(\boldsymbol{u}^{\frac{1+m}{2}}\right)_{r}^{(\theta)}\right|^{2}}{s^{\frac{1+m}{m}}} \mathrm{~d} x \mathrm{~d} t \leq c \int_{Q_{s}^{(\theta)}} \frac{\left|\boldsymbol{u}^{\frac{1+m}{2}}-\left(\boldsymbol{u}^{\frac{1+m}{2}}\right)_{s}^{(\theta)}\right|^{2}}{s^{\frac{1+m}{m}}} \mathrm{~d} x \mathrm{~d} t,
$$

while to term $\mathrm{II}_{2}$ we apply Lemma 3.2 with $\alpha=\frac{1+m}{2 m}$ and Lemma 3.4 and find

$$
\mathrm{II}_{2} \leq c \int_{Q_{s}^{(\theta)}} \frac{\left|\boldsymbol{u}^{\frac{1+m}{2}}-\left(\boldsymbol{u}^{\frac{1+m}{2}}\right)_{r}^{(\theta)}\right|^{2}}{s^{\frac{1+m}{m}}} \mathrm{~d} x \mathrm{~d} t \leq c \int_{Q_{s}^{(\theta)}} \frac{\left|\boldsymbol{u}^{\frac{1+m}{2}}-\left(\boldsymbol{u}^{\frac{1+m}{2}}\right)_{s}^{(\theta)}\right|^{2}}{s^{\frac{1+m}{m}}} \mathrm{~d} x \mathrm{~d} t .
$$

Combining both cases we have

$$
\mathrm{II} \leq \varepsilon \mathcal{R}_{r, s}^{2} \iint_{Q_{s}^{(\theta)}}\left[\left|D \boldsymbol{u}^{m}\right|^{2}+|F|^{2}\right] \mathrm{d} x \mathrm{~d} t+\frac{c \mathcal{R}_{r, s}^{2}}{\varepsilon^{\frac{1-m}{2 m}}} \iint_{Q_{s}^{(\theta)}} \frac{\left|\boldsymbol{u}^{\frac{1+m}{2}}-\left(\boldsymbol{u}^{\frac{1+m}{2}}\right)_{s}^{(\theta)}\right|^{2}}{s^{\frac{1+m}{m}}} \mathrm{~d} x \mathrm{~d} t,
$$

with a constant $c=c(m, \nu, L, K)$. Inserting the estimates for I and II above and applying Lemma 5.1 with $\varepsilon$ replaced by $\varepsilon^{\frac{1+m}{2 m}}$, we find for any $\varepsilon \in(0,1]$ that

$$
\begin{aligned}
\sup _{t \in \Lambda_{r}} & f_{B_{r}^{(\theta)}} \frac{\left|\boldsymbol{u}^{\frac{1+m}{2}}(t)-\left(\boldsymbol{u}^{\frac{1+m}{2}}\right)_{r}^{(\theta)}\right|^{2}}{r^{\frac{1+m}{m}}} \mathrm{~d} x+\int_{Q_{r}^{(\theta)}}\left|D \boldsymbol{u}^{m}\right|^{2} \mathrm{~d} x \mathrm{~d} t \\
\leq & \frac{c \mathcal{R}_{r, s}^{\frac{1+m}{m}}}{\varepsilon^{\frac{1-m}{2 m}}} \iint_{Q_{s}^{(\theta)}} \frac{\left|\boldsymbol{u}^{\frac{1+m}{2}}-\left(\boldsymbol{u}^{\frac{1+m}{2}}\right)_{s}^{(\theta)}\right|^{2}}{s^{\frac{1+m}{m}}} \mathrm{~d} x \mathrm{~d} t \\
& \quad+\varepsilon \mathcal{R}_{r, s}^{2} \int_{Q_{s}^{(\theta)}}\left|D \boldsymbol{u}^{m}\right|^{2} \mathrm{~d} x \mathrm{~d} t+c \mathcal{R}_{r, s}^{2} \iint_{Q_{s}^{(\theta)}}|F|^{2} \mathrm{~d} x \mathrm{~d} t
\end{aligned}
$$




$$
\begin{gathered}
\leq c \varepsilon \mathcal{R}_{r, s}^{\frac{1+m}{m}}\left[\sup _{t \in \Lambda_{s}} f_{B_{s}^{(\theta)}} \frac{\left|\boldsymbol{u}^{\frac{1+m}{2}}(t)-\left(\boldsymbol{u}^{\frac{1+m}{2}}\right)_{s}^{(\theta)}\right|^{2}}{s^{\frac{1+m}{m}}} \mathrm{~d} x+\int_{Q_{s}^{(\theta)}}\left|D \boldsymbol{u}^{m}\right|^{2} \mathrm{~d} x \mathrm{~d} t\right] \\
+\frac{c \mathcal{R}_{r, s}^{\frac{1+m}{m}}}{\varepsilon^{\frac{(1+m)(n+2)}{2 n m}-1}}\left[\left[\iint_{Q_{s}^{(\theta)}}\left|D \boldsymbol{u}^{m}\right|^{2 q} \mathrm{~d} x \mathrm{~d} t\right]^{\frac{1}{q}}+\int_{Q_{s}^{(\theta)}}|F|^{2} \mathrm{~d} x \mathrm{~d} t\right] .
\end{gathered}
$$

Here we choose $\varepsilon=1 /\left[2 c \mathcal{R}_{r, s}^{\frac{1+m}{m}}\right]$. With this choice the first term on the right-hand side tuns into $\frac{1}{2}[\ldots]$, where $[\ldots]$ is the expression from the left-hand side with $r$ replaced by $s$. Moreover, the pre-factor in front of the second term on the right-hand side changes to $\mathcal{R}_{r, s}^{\alpha}$ with $\alpha=\frac{n+2}{2 n}\left(\frac{1+m}{m}\right)^{2}$. To this inequality we apply the Iteration Lemma 3.1 to re-absorb the term $\frac{1}{2}[\ldots]$ (with radius $s$ ) from the right-hand side into the left. This leads to the claimed reverse Hölder type inequality

$$
\begin{gathered}
\sup _{t \in \Lambda_{\varrho}} f_{B_{\varrho}^{(\theta)}} \frac{\left|\boldsymbol{u}^{\frac{1+m}{2}}(t)-\left(\boldsymbol{u}^{\frac{1+m}{2}}\right)_{\varrho}^{(\theta)}\right|^{2}}{\varrho^{\frac{1+m}{m}}} \mathrm{~d} x+\int_{Q_{\varrho}^{(\theta)}}\left|D \boldsymbol{u}^{m}\right|^{2} \mathrm{~d} x \mathrm{~d} t \\
\leq c\left[\iint_{Q_{2 \varrho}^{(\theta)}}\left|D \boldsymbol{u}^{m}\right|^{2 q} \mathrm{~d} x \mathrm{~d} t\right]^{\frac{1}{q}}+c \iint_{Q_{2 \varrho}^{(\theta)}}|F|^{2} \mathrm{~d} x \mathrm{~d} t,
\end{gathered}
$$

and finishes the proof.

At the end of this section we provide a technical auxiliary result, which essentially is a direct consequence of Lemma 5.1 and the energy estimate.

Lemma 6.2. Let $m \in\left(m_{c}, 1\right]$ and $u$ be a weak solution to (2.2) in $\Omega_{T}$ in the sense of Definition 2.1. Then, on any cylinder $Q_{2 \varrho}^{(\theta)}\left(z_{o}\right) \subseteq \Omega_{T}$ with $\varrho, \theta>0$ satisfying (6.1) and (6.2) 1 with $K=1$, we have

$$
\theta^{m} \leq \frac{1}{\sqrt{2}}\left[\int_{Q_{\varrho / 2}^{(\theta)}\left(z_{o}\right)} \frac{|u|^{1+m}}{(\varrho / 2)^{\frac{1+m}{m}}} \mathrm{~d} x \mathrm{~d} t\right]^{\frac{1}{2}}+c\left[\int_{Q_{Q_{\varrho}\left(z_{o}\right)}^{(\theta)}}\left[\left|D \boldsymbol{u}^{m}\right|^{2}+|F|^{2}\right] \mathrm{d} x \mathrm{~d} t\right]^{\frac{1}{2}}
$$

where $c=c(n, m, \nu, L)$.

Proof. We omit the reference to the center $z_{o}$ in the notation. We use $(6.2)_{1}$ with $K=1$, Minkowski's inequality and Lemma 3.4 to deduce

$$
\begin{aligned}
\theta^{m} & \leq\left[\iint_{Q_{\varrho}^{(\theta)}} \frac{|u|^{1+m}}{\varrho^{\frac{1+m}{m}}} \mathrm{~d} x \mathrm{~d} t\right]^{\frac{1}{2}} \\
& \leq\left[\iint_{Q_{\varrho}^{(\theta)}} \frac{\left|\boldsymbol{u}^{\frac{1+m}{2}}-\left(\boldsymbol{u}^{\frac{1+m}{2}}\right)_{\varrho / 2}^{(\theta)}\right|^{2}}{\varrho^{\frac{1+m}{m}}} \mathrm{~d} x \mathrm{~d} t\right]^{\frac{1}{2}}+\frac{\left|\left(\boldsymbol{u}^{\frac{1+m}{2}}\right)_{\varrho / 2}^{(\theta)}\right|}{\varrho^{\frac{1+m}{2 m}}} \\
& \leq c\left[\iint_{Q_{\varrho}^{(\theta)}} \frac{\left|\boldsymbol{u}^{\frac{1+m}{2}}-\left(\boldsymbol{u}^{\frac{1+m}{2}}\right)_{\varrho}^{(\theta)}\right|^{2}}{\varrho^{\frac{1+m}{m}}} \mathrm{~d} x \mathrm{~d} t\right]^{\frac{1}{2}}+\left[\iint_{Q_{\varrho / 2}^{(\theta)}} \frac{|u|^{1+m}}{\varrho^{\frac{1+m}{m}}} \mathrm{~d} x \mathrm{~d} t\right]^{\frac{1}{2}} .
\end{aligned}
$$

We estimate the first term on the right with Lemma 5.1 and Hölder's inequality and get

$$
\begin{aligned}
\int_{Q_{\varrho}^{(\theta)}} \frac{\left|\boldsymbol{u}^{\frac{1+m}{2}}-\left(\boldsymbol{u}^{\frac{1+m}{2}}\right)_{\varrho}^{(\theta)}\right|^{2}}{\varrho^{\frac{1+m}{m}} \mathrm{~d} x \mathrm{~d} t \leq} & \varepsilon \sup _{t \in \Lambda_{\varrho}} \int_{B_{\varrho}^{(\theta)}} \frac{\left|\boldsymbol{u}^{\frac{1+m}{2}}(t)-\left(\boldsymbol{u}^{\frac{1+m}{2}}\right) \varrho^{(\theta)}\right|^{2}}{\varrho^{\frac{1+m}{m}}} \mathrm{~d} x \\
& +\frac{c}{\varepsilon^{\frac{2}{n}}} \iint_{Q_{\varrho}^{(\theta)}}\left[\left|D \boldsymbol{u}^{m}\right|^{2}+|F|^{2}\right] \mathrm{d} x \mathrm{~d} t,
\end{aligned}
$$

for a constant $c=c(n, m, L)$ and an arbitrary $\varepsilon \in(0,1]$. In order to bound the sup-term appearing in the last estimate, we apply the energy estimate from Lemma 4.1, combined 
with Lemma 3.4 with $a=0$, Hölder's inequality and hypothesis (6.1), with the result

$$
\begin{aligned}
\sup _{t \in \Lambda_{\varrho}} & f_{B_{\varrho}^{(\theta)}} \frac{\left|\boldsymbol{u}^{\frac{1+m}{2}}(t)-\left(\boldsymbol{u}^{\frac{1+m}{2}}\right)_{\varrho}^{(\theta)}\right|^{2}}{\varrho^{\frac{1+m}{m}}} \mathrm{~d} x \\
& \leq c \int_{Q_{2 \varrho}^{(\theta)}}\left[\frac{|u|^{1+m}}{\varrho^{\frac{1+m}{m}}}+\frac{|u|^{2 m}+\left|\left(\boldsymbol{u}^{\frac{1+m}{2}}\right) \varrho^{(\theta)}\right|^{\frac{4 m}{1+m}}}{\theta^{\frac{2 m(m-1)}{1+m}} \varrho^{2}}+|F|^{2}\right] \mathrm{d} x \mathrm{~d} t \\
& \leq c \int_{Q_{2 \varrho}^{(\theta)}} \frac{|u|^{1+m}}{\varrho^{\frac{1+m}{m}}} \mathrm{~d} x \mathrm{~d} t+c\left[\int_{Q_{2 \varrho}^{(\theta)}} \frac{\theta^{1-m}|u|^{1+m}}{\varrho^{\frac{1+m}{m}}} \mathrm{~d} x \mathrm{~d} t\right]^{\frac{2 m}{1+m}}+c \int_{Q_{2 \varrho}^{(\theta)}}|F|^{2} \mathrm{~d} x \mathrm{~d} t \\
& \leq c \theta^{2 m}+c \int_{Q_{2 \varrho}^{(\theta)}}|F|^{2} \mathrm{~d} x \mathrm{~d} t,
\end{aligned}
$$

where $c=c(m, \nu, L)$. Joining the preceding inequalities leads us to

$$
\theta^{m} \leq c \sqrt{\varepsilon} \theta^{m}+\left[\iint_{Q_{\varrho / 2}^{(\theta)}} \frac{|u|^{1+m}}{\varrho^{\frac{1+m}{m}}} \mathrm{~d} x \mathrm{~d} t\right]^{\frac{1}{2}}+\frac{c}{\varepsilon^{\frac{1}{n}}}\left[\iint_{Q_{2 \varrho}^{(\theta)}}\left[\left|D \boldsymbol{u}^{m}\right|^{2}+|F|^{2}\right] \mathrm{d} x \mathrm{~d} t\right]^{\frac{1}{2}}
$$

After choosing $\varepsilon=\varepsilon(n, m, L) \in(0,1]$ so small that $c \sqrt{\varepsilon} \leq 1-2^{-\frac{1}{2 m}}$, we can re-absorb the first term of the right-hand side into the left. In this way, we obtain

$$
\theta^{m} \leq 2^{\frac{1}{2 m}}\left[\int_{Q_{\varrho / 2}^{(\theta)}} \frac{|u|^{1+m}}{\varrho^{\frac{1+m}{m}}} \mathrm{~d} x \mathrm{~d} t\right]^{\frac{1}{2}}+c\left[\int_{Q_{2 \varrho}^{(\theta)}}\left[\left|D \boldsymbol{u}^{m}\right|^{2}+|F|^{2}\right] \mathrm{d} x \mathrm{~d} t\right]
$$

which proves the asserted inequality.

\section{PROOF OF THE HIGHER INTEGRABILITY}

We consider a fixed cylinder

$$
Q_{8 R}\left(y_{o}, \tau_{o}\right) \equiv B_{8 R}\left(y_{o}\right) \times\left(\tau_{o}-(8 R)^{\frac{1+m}{m}}, \tau_{o}+(8 R)^{\frac{1+m}{m}}\right) \subseteq \Omega_{T}
$$

with $R>0$. Again, we omit the center in the notation and write $Q_{\varrho}:=Q_{\varrho}\left(y_{o}, \tau_{o}\right)$ for short, for any radius $\varrho \in(0,8 R]$. We consider a parameter

$$
\lambda_{o} \geq 1+\left[\int_{Q_{4 R}} \frac{|u|^{1+m}}{(4 R)^{\frac{1+m}{m}}} \mathrm{~d} x \mathrm{~d} t\right]^{\frac{d}{2 m}},
$$

which will be fixed later. Recall that the scaling deficit $d$ is defined in (2.7). We again point out that the assumption $m>m_{c}$ ensures $d>0$. Furthermore, for $n \geq 2$ the scaling deficit blows up when $m \downarrow m_{c}$. For $z_{o} \in Q_{2 R}, \varrho \in(0, R]$, and $\theta \geq 1$, we consider space-time cylinders $Q_{\varrho}^{(\theta)}\left(z_{o}\right)$ as defined in (2.1). Note that these cylinders depend monotonically on $\theta$ in the sense that $Q_{\varrho}^{\left(\theta_{2}\right)}\left(z_{o}\right) \subset Q_{\varrho}^{\left(\theta_{1}\right)}\left(z_{o}\right)$ whenever $1 \leq \theta_{1}<\theta_{2}$, and that $Q_{\varrho}^{(\theta)}\left(z_{o}\right) \subset Q_{4 R}$ for $z_{o} \in Q_{2 R}, \varrho \in(0, R]$, and $\theta \geq 1$.

7.1. Construction of a non-uniform system of cylinders. The following construction of a non-uniform system of cylinders is similar to the one in [11, 19]. Let $z_{o} \in Q_{2 R}$. For a radius $\varrho \in(0, R]$ we define

$$
\widetilde{\theta}_{\varrho} \equiv \widetilde{\theta}_{z_{o} ; \varrho}:=\inf \left\{\theta \in\left[\lambda_{o}, \infty\right): \frac{1}{\left|Q_{\varrho}\right|} \iint_{Q_{\varrho}^{(\theta)}\left(z_{o}\right)} \frac{|u|^{1+m}}{\varrho^{\frac{1+m}{m}}} \mathrm{~d} x \mathrm{~d} t \leq \theta^{\frac{2 m}{d}}\right\} .
$$

We note that $\widetilde{\theta}_{\varrho}$ is well defined, since the infimum in the definition is taken over a nonempty set. In fact, in the limit $\theta \rightarrow \infty$ the integral on the left-hand side converges to zero (and is constant in the case $m=1$, respectively), while the right-hand side grows with 
speed $\theta^{\frac{2 m}{d}}$. The choice of the exponent on the right-hand side becomes more clear after taking means in the integral condition, since then the condition takes the form

$$
\int_{Q_{\varrho}^{(\theta)}\left(z_{o}\right)} \frac{|u|^{1+m}}{\varrho^{\frac{1+m}{m}}} \mathrm{~d} x \mathrm{~d} t \leq \theta^{2 m}
$$

compare Sections 5 and 6 . As an immediate consequence of the definition of $\widetilde{\theta}_{\varrho}$, we either have

or

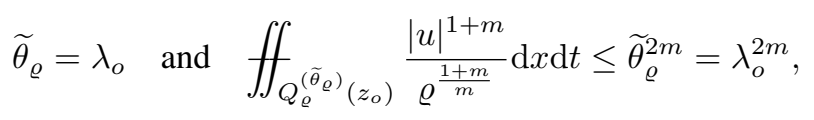

$$
\widetilde{\theta}_{\varrho}>\lambda_{o} \quad \text { and } \quad \iint_{Q_{\varrho}^{\left(\tilde{\theta}_{\varrho}\right)}\left(z_{o}\right)} \frac{|u|^{1+m}}{\varrho^{\frac{1+m}{m}}} \mathrm{~d} x \mathrm{~d} t=\widetilde{\theta}_{\varrho}^{2 m} .
$$

In the case $\varrho=R$, we have $\widetilde{\theta}_{R} \geq \lambda_{o} \geq 1$. Moreover, in the case $\widetilde{\theta}_{R}>\lambda_{o}$, property (7.2), the inclusion $Q_{R}^{\left(\widetilde{\theta}_{R}\right)}\left(z_{o}\right) \subset Q_{4 R}$ and (7.1) yield that

$$
\begin{aligned}
\widetilde{\theta}_{R}^{\frac{2 m}{d}} & =\frac{1}{\left|Q_{R}\right|} \iint_{Q_{R}^{\left(\widetilde{\theta}_{R}\right)}\left(z_{o}\right)} \frac{|u|^{1+m}}{R^{\frac{1+m}{m}}} \mathrm{~d} x \mathrm{~d} t \\
& \leq \frac{4^{\frac{1+m}{m}}}{\left|Q_{R}\right|} \iint_{Q_{4 R}} \frac{|u|^{1+m}}{(4 R)^{\frac{1+m}{m}}} \mathrm{~d} x \mathrm{~d} t \leq 4^{n+2+\frac{2}{m}} \lambda_{o}^{\frac{2 m}{d}},
\end{aligned}
$$

from which we infer the bound

$$
\widetilde{\theta}_{R} \leq 4^{\frac{d}{2 m}\left(n+2+\frac{2}{m}\right)} \lambda_{o}
$$

Our next goal is to prove the continuity of the mapping $(0, R] \ni \varrho \mapsto \widetilde{\theta}_{\varrho}$. For $\varrho \in(0, R]$ and $\varepsilon>0$, we abbreviate $\theta_{+}:=\widetilde{\theta}_{\varrho}+\varepsilon$. We first observe that there exists $\delta=\delta(\varepsilon, \varrho)>0$ such that for all radii $r \in(0, R]$ with $|r-\varrho|<\delta$ there holds

$$
\frac{1}{\left|Q_{r}\right|} \iint_{Q_{r}^{\left(\theta_{+}\right)}\left(z_{o}\right)} \frac{|u|^{1+m}}{r^{\frac{1+m}{m}}} \mathrm{~d} x \mathrm{~d} t<\theta_{+}^{\frac{2 m}{d}} .
$$

In fact, if $r=\varrho$, this is a consequence of the definition of $\widetilde{\theta}_{\varrho}$, since $\theta_{+}^{\frac{2 m}{d}}>\widetilde{\theta}_{\varrho}^{\frac{2 m}{d}}$. By the absolute continuity of the integral, the inequality (7.4) continues to hold for radii $r$ sufficiently close to $\varrho$. Hence, the definition of $\widetilde{\theta}_{r}$ implies that $\widetilde{\theta}_{r}<\theta_{+}=\widetilde{\theta}_{\varrho}+\varepsilon$, provided $|r-\varrho|<\delta$. For the corresponding lower bound $\widetilde{\theta}_{r}>\theta_{-}:=\widetilde{\theta}_{\varrho}-\varepsilon$, we proceed similarly. First, we note that we can assume $\theta_{-} \geq \lambda_{o}$ and hence $\widetilde{\theta}_{\varrho}>\lambda_{o}$, since otherwise the claim immediately follows from the property $\widetilde{\theta}_{r} \geq \lambda_{o}$. Now, we claim that

$$
\frac{1}{\left|Q_{r}\right|} \iint_{Q_{r}^{\left(\theta_{-}\right)}\left(z_{o}\right)} \frac{|u|^{1+m}}{r^{\frac{1+m}{m}}} \mathrm{~d} x \mathrm{~d} t>\theta_{-}^{\frac{2 m}{d}}
$$

for all $r \in(0, R]$ with $|r-\varrho|<\delta$, after diminishing $\delta=\delta(\varepsilon, \varrho)>0$ if necessary. Again, we first consider the case $r=\varrho$, in which the claim follows from the definition of $\widetilde{\theta}_{\varrho}$. In fact, if the claim did not hold, we would arrive at the contradiction $\widetilde{\theta}_{\varrho} \leq \theta_{-}$. Now, for radii $r$ with $|r-\varrho|<\delta$ the assertion follows from the continuous dependence of the left-hand side upon $r$. Having established (7.5), we can conclude from the definition of $\widetilde{\theta}_{r}$ that $\widetilde{\theta}_{r}>\theta_{-}=\widetilde{\theta}_{\varrho}-\varepsilon$. Altogether we have shown that $\widetilde{\theta}_{\varrho}-\varepsilon<\widetilde{\theta}_{r}<\widetilde{\theta}_{\varrho}+\varepsilon$ for all radii $r \in(0, R]$ with $|r-\varrho|<\delta$, which completes the proof of the continuity of $(0, R] \ni \varrho \mapsto \widetilde{\theta}_{\varrho}$.

Unfortunately, the mapping $(0, R] \ni \varrho \rightarrow \widetilde{\theta}_{\varrho}$ might not be decreasing. For this reason we work with a modified version of $\widetilde{\theta}_{\varrho}$, which we denote by $\theta_{\varrho}$. This modification is done by a rising sun type construction. More precisely, we define

$$
\theta_{\varrho} \equiv \theta_{z_{o} ; \varrho}:=\max _{r \in[\varrho, R]} \tilde{\theta}_{z_{o} ; r}
$$


As an immediate consequence of the construction, the mapping $(0, R] \ni \varrho \mapsto \theta_{\varrho}$ is continuous and monotonically decreasing. In general, the modified cylinders $Q_{\varrho}^{\left(\theta_{\varrho}\right)}\left(z_{o}\right)$ cannot be expected to be intrinsic in the sense of (7.2). However, we can show that the cylinders $Q_{s}^{\left(\theta_{\varrho}\right)}\left(z_{o}\right)$ are sub-intrinsic for all radii $s \geq \varrho$. More precisely, we have

$$
\int_{Q_{s}^{\left(\theta_{\varrho}\right)}\left(z_{o}\right)} \frac{|u|^{1+m}}{s^{\frac{1+m}{m}}} \mathrm{~d} x \mathrm{~d} t \leq \theta_{\varrho}^{2 m} \quad \text { for any } 0<\varrho \leq s \leq R .
$$

For the proof of this inequality, we use the chain of inequalities $\widetilde{\theta}_{s} \leq \theta_{s} \leq \theta_{\varrho}$, which implies $Q_{s}^{\left(\theta_{\varrho}\right)}\left(z_{o}\right) \subseteq Q_{s}^{\left(\widetilde{\theta}_{s}\right)}\left(z_{o}\right)$, and the fact that the latter cylinder is sub-intrinsic. In this way, we deduce

$$
\begin{aligned}
\iint_{Q_{s}^{\left(\theta_{\varrho}\right)}\left(z_{o}\right)} \frac{|u|^{1+m}}{s^{\frac{1+m}{m}}} \mathrm{~d} x \mathrm{~d} t & \leq\left(\frac{\theta_{\varrho}}{\widetilde{\theta}_{s}}\right)^{2 m-\frac{2 m}{d}} \iint_{Q_{s}^{\left(\tilde{\theta}_{s}\right)}\left(z_{o}\right)} \frac{|u|^{1+m}}{s^{\frac{1+m}{m}}} \mathrm{~d} x \mathrm{~d} t \\
& \leq\left(\frac{\theta_{\varrho}}{\widetilde{\theta}_{s}}\right)^{2 m-\frac{2 m}{d}} \widetilde{\theta}_{s}^{2 m}=\theta_{\varrho}^{2 m-\frac{2 m}{d}} \widetilde{\theta}_{s}^{\frac{2 m}{d}} \leq \theta_{\varrho}^{2 m}
\end{aligned}
$$

which is exactly assertion (7.6). Next, we define

$$
\widetilde{\varrho}:=\left\{\begin{array}{cl}
R, & \text { if } \theta_{\varrho}=\lambda_{o}, \\
\min \left\{s \in[\varrho, R]: \theta_{s}=\widetilde{\theta}_{s}\right\}, & \text { if } \theta_{\varrho}>\lambda_{o} .
\end{array}\right.
$$

By definition, for any $s \in[\varrho, \widetilde{\varrho}]$ we have $\theta_{s}=\widetilde{\theta}_{\widetilde{\varrho}}$. Our next goal is the proof of the upper bound

$$
\theta_{\varrho} \leq\left(\frac{s}{\varrho}\right)^{\frac{d}{2 m}\left(n+2+\frac{2}{m}\right)} \theta_{s} \quad \text { for any } s \in(\varrho, R] .
$$

In the case $\theta_{\varrho}=\lambda_{o}$ this is immediate since $\theta_{s} \geq \lambda_{o}$. Another easy case is that of radii $s \in(\varrho, \widetilde{\varrho}]$, since then we have $\theta_{s}=\widetilde{\theta}_{\widetilde{\varrho}}=\theta_{\varrho}$. Therefore, it only remains to prove (7.8) for the case $\theta_{\varrho}>\lambda_{o}$ and radii $s \in(\widetilde{\varrho}, R]$. To this end, we use the monotonicity of $\varrho \mapsto \theta_{\varrho}$, (7.2) and (7.6) to conclude

$$
\begin{aligned}
\theta_{\varrho}^{\frac{2 m}{d}} & =\widetilde{\theta}_{\widetilde{\varrho}^{d}}^{\frac{2 m}{d}}=\frac{1}{\left|Q_{\widetilde{\varrho}}\right|} \iint_{Q_{\widetilde{\varrho}}^{(\theta \widetilde{\varrho})}\left(z_{o}\right)} \frac{|u|^{1+m}}{\widetilde{\varrho}^{\frac{1+m}{m}}} \mathrm{~d} x \mathrm{~d} t \\
& \leq\left(\frac{s}{\widetilde{\varrho}}\right)^{n+2+\frac{2}{m}} \frac{1}{\left|Q_{s}\right|} \iint_{Q_{s}^{\left(\theta_{s}\right)}\left(z_{o}\right)} \frac{|u|^{1+m}}{s^{\frac{1+m}{m}}} \mathrm{~d} x \mathrm{~d} t \leq\left(\frac{s}{\varrho}\right)^{n+2+\frac{2}{m}} \theta_{s}^{\frac{2 m}{d}} .
\end{aligned}
$$

This yields the claim (7.8) also in the remaining case. We now apply (7.8) with $s=R$. Using moreover the fact $\theta_{R}=\widetilde{\theta}_{R}$ and estimate (7.3) for $\widetilde{\theta}_{R}$, we deduce

$$
\theta_{\varrho} \leq\left(\frac{R}{\varrho}\right)^{\frac{d}{2 m}\left(n+2+\frac{2}{m}\right)} \theta_{R} \leq\left(\frac{4 R}{\varrho}\right)^{\frac{d}{2 m}\left(n+2+\frac{2}{m}\right)} \lambda_{o}
$$

for every $\varrho \in(0, R]$. In summary, for every $z_{o} \in Q_{2 R}$, we have constructed a system of concentric sub-intrinsic cylinders $Q_{\varrho}^{\left(\theta_{z_{o} ; \varrho}\right)}\left(z_{o}\right)$ with radii $\varrho \in(0, R]$. As a consequence of the monotonicity of $\varrho \mapsto \theta_{z_{o} ; \varrho}$, these cylinders are nested in the sense that

$$
Q_{r}^{\left(\theta_{z_{o} ; r}\right)}\left(z_{o}\right) \subset Q_{s}^{\left(\theta_{z_{o} ; s}\right)}\left(z_{o}\right) \text { whenever } 0<r<s \leq R .
$$

However, keep in mind that in general these cylinders are not intrinsic but only subintrinsic. 
7.2. Covering property. Our next goal is to establish the following Vitali type covering property for the cylinders constructed in the last section.

Lemma 7.1. There exists a constant $\hat{c}=\hat{c}(n, m) \geq 20$ such that, whenever $\mathcal{F}$ is a collection of cylinders $Q_{4 r}^{\left(\theta_{z ; r}\right)}(z)$, where $Q_{r}^{\left(\theta_{z ; r}\right)}(z)$ is a cylinder of the form constructed in Section 7.1 with radius $r \in\left(0, \frac{R}{\hat{c}}\right)$, then there exists a countable subfamily $\mathcal{G}$ of disjoint cylinders in $\mathcal{F}$ such that

$$
\bigcup_{Q \in \mathcal{F}} Q \subseteq \bigcup_{Q \in \mathcal{G}} \widehat{Q}
$$

where $\widehat{Q}$ denotes the $\frac{\hat{c}}{4}$-times enlarged cylinder $Q$, i.e. if $Q=Q_{4 r}^{\left(\theta_{z ; r}\right)}(z)$, then $\widehat{Q}=$ $Q_{\hat{c} r}^{\left(\theta_{z ; r}\right)}(z)$.

Proof. For $j \in \mathbb{N}$ we subdivide $\mathcal{F}$ into the subfamilies

$$
\mathcal{F}_{j}:=\left\{Q_{4 r}^{\left(\theta_{z ; r}\right)}(z) \in \mathcal{F}: \frac{R}{2^{j} \hat{c}}<r \leq \frac{R}{2^{j-1} \hat{c}}\right\} .
$$

Then, we choose finite subfamilies $\mathcal{G}_{j} \subset \mathcal{F}_{j}$ according to the following scheme. We start by choosing $\mathcal{G}_{1}$ as an arbitrary maximal disjoint collection of cylinders in $\mathcal{F}_{1}$. The subfamily $\mathcal{G}_{1}$ is finite, since (7.9) and the definition of $\mathcal{F}_{1}$ imply a lower bound on the volume of each cylinder in $\mathcal{G}_{1}$. Now, assuming that the subfamilies $\mathcal{G}_{1}, \mathcal{G}_{2}, \ldots, \mathcal{G}_{k-1}$ have already been constructed for some integer $k \geq 2$, we choose $\mathcal{G}_{k}$ to be any maximal disjoint subcollection of

$$
\left\{Q \in \mathcal{F}_{k}: Q \cap Q^{*}=\emptyset \text { for any } Q^{*} \in \bigcup_{j=1}^{k-1} \mathcal{G}_{j}\right\} .
$$

For the same reason as above, the collection $\mathcal{G}_{k}$ is finite. Hence, the family

$$
\mathcal{G}:=\bigcup_{j=1}^{\infty} \mathcal{G}_{j} \subseteq \mathcal{F}
$$

defines a countable collection of disjoint cylinders. It remains to prove that for each cylin$\operatorname{der} Q \in \mathcal{F}$ there exists a cylinder $Q^{*} \in \mathcal{G}$ with $Q \subset \widehat{Q}^{*}$. To this end, we fix a cylinder $Q=Q_{4 r}^{\left(\theta_{z ; r}\right)}(z) \in \mathcal{F}$. Let $j \in \mathbb{N}$ be such that $Q \in \mathcal{F}_{j}$. The maximality of $\mathcal{G}_{j}$ ensures the existence of a cylinder $Q^{*}=Q_{4 r_{*}}^{\left(\theta_{z_{*} ; r_{*}}\right)}\left(z_{*}\right) \in \bigcup_{i=1}^{j} \mathcal{G}_{i}$ with $Q \cap Q^{*} \neq \emptyset$. We will show that this cylinder has the desired property $Q \subset \widehat{Q}^{*}$. First, we observe that the properties $r \leq \frac{R}{2^{j-1} \hat{c}}$ and $r_{*}>\frac{R}{2^{j} \hat{c}}$ imply $r \leq 2 r_{*}$, which ensures $\Lambda_{4 r}(t) \subseteq \Lambda_{20 r_{*}}\left(t_{*}\right)$. For the proof of the corresponding spatial inclusion $B_{4 r}^{\left(\theta_{z, r}\right)}(x) \subseteq B_{\hat{c} r_{*}}^{\left(\theta_{z_{*}, r_{*}}\right)}\left(x_{*}\right)$, we first shall derive the bound

$$
\theta_{z_{*} ; r_{*}} \leq 52^{\frac{d}{2 m}\left(n+2+\frac{2}{m}\right)} \theta_{z ; r} .
$$

We recall the definition (7.7) of the radius $\widetilde{r}_{*} \in\left[r_{*}, R\right]$ which is associated to the cylinder $Q_{r_{*}}^{\left(\theta_{z_{*} ; r_{*}}\right)}\left(z_{*}\right)$. According to the definition, we either have that $Q_{\widetilde{r}_{*}}^{\left(\theta_{z_{*} ; r_{*}}\right)}\left(z_{*}\right)$ is intrinsic or that $\widetilde{r}_{*}=R$ and $\theta_{z_{*} ; r_{*}}=\lambda_{o}$. In the second alternative, the claim (7.11) is immediate, since

$$
\theta_{z_{*} ; r_{*}}=\lambda_{o} \leq \theta_{z ; r} .
$$

Therefore, it remains to consider the case that $Q_{\widetilde{r}_{*}}^{\left(\theta_{z_{*} ; r_{*}}\right)}\left(z_{*}\right)$ is intrinsic in the sense that

$$
\theta_{z_{*} ; r_{*}}^{\frac{2 m}{d}}=\frac{1}{\left|Q_{\widetilde{r}_{*}}\right|} \iint_{Q_{\widetilde{r}_{*}}^{\left(\theta_{*} ; r_{*}\right)}\left(z_{*}\right)} \frac{|u|^{1+m}}{\widetilde{r}_{*}^{\frac{1+m}{m}}} \mathrm{~d} x \mathrm{~d} t .
$$


We distinguish between the cases $\widetilde{r}_{*} \leq \frac{R}{\mu}$ and $\widetilde{r}_{*}>\frac{R}{\mu}$, where $\mu:=13$. We start with the latter case. Using (7.12) and the definition of $\lambda_{o}$ and $\theta_{z ; r}$, we estimate

$$
\begin{aligned}
\theta_{z_{*} ; r_{*}}^{\frac{2 m}{d}} & \leq\left(\frac{4 R}{\widetilde{r}_{*}}\right)^{\frac{1+m}{m}} \frac{1}{\left|Q_{\widetilde{r}_{*}}\right|} \iint_{Q_{4 R}} \frac{|u|^{1+m}}{(4 R)^{\frac{1+m}{m}}} \mathrm{~d} x \mathrm{~d} t \\
& \leq\left(\frac{4 R}{\widetilde{r}_{*}}\right)^{n+2+\frac{2}{m}} \lambda_{o}^{\frac{2 m}{d}} \\
& \leq(4 \mu)^{n+2+\frac{2}{m}} \theta_{z ; r}^{\frac{2 m}{d}},
\end{aligned}
$$

which can be rewritten in the form

$$
\theta_{z_{*} ; r_{*}} \leq(4 \mu)^{\frac{d}{2 m}\left(n+2+\frac{2}{m}\right)} \theta_{z ; r} .
$$

This yields (7.12) in the second case, and it only remains to consider the first case $\widetilde{r}_{*} \leq \frac{R}{\mu}$. Here, the key step is to prove the inclusion

$$
Q_{\widetilde{r}_{*}}^{\left(\theta_{z_{*} ; r_{*}}\right)}\left(z_{*}\right) \subseteq Q_{\mu \widetilde{r}_{*}}^{\left(\theta_{z ; \tilde{r}_{*}}\right)}(z)
$$

We first observe that $\widetilde{r}_{*} \geq r_{*}$ and $\left|t-t_{*}\right|<(4 r)^{\frac{1+m}{m}}+\left(4 r_{*}\right)^{\frac{1+m}{m}} \leq\left(12 r_{*}\right)^{\frac{1+m}{m}}$ implies $\Lambda_{\widetilde{r}_{*}}\left(t_{*}\right) \subseteq \Lambda_{\mu \widetilde{r}_{*}}(t)$. In addition, we have

$$
\left|x-x_{*}\right| \leq \theta_{z ; r}^{\frac{m(m-1)}{1+m}} 4 r+\theta_{z_{*} ; r_{*}}^{\frac{m(m-1)}{1+m}} 4 r_{*} .
$$

At this point, we may assume that $\theta_{z ; r} \leq \theta_{z_{*} ; r_{*}}$, since (7.11) clearly is satisfied in the alternative case. Then, the monotonicity of $\varrho \mapsto \theta_{z ; \varrho}$ and $r \leq 2 r_{*} \leq 2 \widetilde{r}_{*} \leq \mu \widetilde{r}_{*}$ imply

$$
\theta_{z_{*} ; r_{*}} \geq \theta_{z ; r} \geq \theta_{z ; \mu \widetilde{r}_{*}} .
$$

Combining this with (7.14), we conclude that

$$
\theta_{z_{*} ; r_{*}}^{\frac{m(m-1)}{1+m}} \widetilde{r}_{*}+\left|x-x_{*}\right| \leq \theta_{z_{*} ; r_{*}}^{\frac{m(m-1)}{1+m}} 5 \widetilde{r}_{*}+\theta_{z ; r}^{\frac{m(m-1)}{1+m}} 4 r \leq \theta_{z ; \mu \widetilde{r}_{*}}^{\frac{m(m-1)}{1+m}} \mu \widetilde{r}_{*},
$$

from which we deduce the inclusion

$$
B_{\widetilde{r}_{*}}^{\left(\theta_{z_{*} ; r_{*}}\right)}\left(x_{*}\right) \subseteq B_{\mu \tilde{r}_{*}}^{\left(\theta_{z ; \mu \tilde{r}_{*}}\right)}(x) .
$$

This completes the proof of (7.13).

Using (7.12), (7.13), and (7.6) with $\varrho=s=\mu \tilde{r}_{*}$, we estimate

$$
\theta_{z_{*} ; r_{*}}^{\frac{2 m}{d}} \leq \frac{\mu^{\frac{1+m}{m}}}{\left|Q_{\widetilde{r}_{*}}\right|} \iint_{Q_{\left.\mu \tilde{r}_{*} ; \mu \tilde{r}_{*}\right)}^{\left(\theta^{\prime}\right)}} \frac{|u|^{1+m}}{\left(\mu \tilde{r}_{*}\right)^{\frac{1+m}{m}}} \mathrm{~d} x \mathrm{~d} t \leq \mu^{n+2+\frac{2}{m}} \theta_{z ; r}^{\frac{2 m}{d}}
$$

which implies

$$
\theta_{z_{*} ; r_{*}} \leq \mu^{\frac{d}{2 m}\left(n+2+\frac{2}{m}\right)} \theta_{z ; r} .
$$

This yields (7.11) also in the last case.

Having established (7.11), it remains to prove the inclusion $Q_{4 r}^{\left(\theta_{z ; r}\right)}(z) \subseteq Q_{\hat{c} r_{*}}^{\left(\theta_{z_{*} ; r_{*}}\right)}\left(z_{*}\right)$, which will complete the proof of the Vitali covering property. First, we note that for any choice of $\hat{c}$ with $\hat{c} \geq 20$, we have $\Lambda_{4 r}(t) \subseteq \Lambda_{\hat{c} r_{*}}\left(t_{*}\right)$. Moreover, from the facts (7.14), $r \leq 2 r_{*}$, and (7.11) we conclude

$$
\begin{aligned}
\theta_{z ; r}^{\frac{m(m-1)}{1+m}} 4 r+\left|x-x_{*}\right| & \leq 2 \theta_{z ; r}^{\frac{m(m-1)}{1+m}} 4 r+\theta_{z_{*} ; r_{*}}^{\frac{m(m-1)}{1+m}} 4 r_{*} \\
& \leq 4\left[4 \cdot 52^{\frac{d(1-m)[m(n+2)+2]}{2 m(1+m)}}+1\right] \theta_{z_{*} ; r_{*}}^{\frac{m(m-1)}{1+m}} r_{*} \\
& \leq \theta_{z_{*} ; r_{*}}^{\frac{m(m-1)}{1+m}} \hat{c} r_{*},
\end{aligned}
$$

for a suitable choice of the constant $\hat{c}=\hat{c}(n, m) \geq 20$. This implies the spatial inclusion $B_{4 r}^{\left(\theta_{z ; r}\right)}(x) \subseteq B_{\hat{c} r_{*}}^{\left(\theta_{z_{*} ; r_{*}}\right)}\left(x_{*}\right)$, which is the remaining piece of information to conclude that

$$
Q=Q_{4 r}^{\left(\theta_{z ; r}\right)}(z) \subseteq Q_{\hat{c} r_{*}}^{\left(\theta_{z_{*} ; r_{*}}\right)}\left(z_{*}\right)=\widehat{Q}^{*} .
$$


Thereby we have established the inclusion (7.10), which yields the desired Vitali type covering property.

7.3. Stopping time argument. Now, we fix the parameter $\lambda_{o}$ by letting

$$
\lambda_{o}:=1+\left[\iint_{Q_{4 R}}\left[\frac{|u|^{1+m}}{(4 R)^{\frac{1+m}{m}}}+\left|D \boldsymbol{u}^{m}\right|^{2}+|F|^{2}\right] \mathrm{d} x \mathrm{~d} t\right]^{\frac{d}{2 m}} .
$$

For $\lambda>\lambda_{o}$ and $r \in(0,2 R]$, we define the super-level set of the function $\left|D \boldsymbol{u}^{m}\right|$ by

$$
\boldsymbol{E}(r, \lambda):=\left\{z \in Q_{r}: z \text { is a Lebesgue point of }\left|D \boldsymbol{u}^{m}\right| \text { and }\left|D \boldsymbol{u}^{m}\right|(z)>\lambda^{m}\right\} .
$$

In the definition of $\boldsymbol{E}(r, \lambda)$, the notion of Lebesgue points is to be understood with regard to the system of cylinders constructed in Section 7.1. We point out that also with respect to these cylinders, $\mathcal{L}^{n+1}$-a.e. point is a Lebesgue point. This follows from [10, 2.9.1], since we already have verified the Vitali type covering property in Lemma 7.1. Now, we fix radii $R \leq R_{1}<R_{2} \leq 2 R$. Note that for any $z_{o} \in Q_{R_{1}}, \kappa \geq 1$ and $\varrho \in\left(0, R_{2}-R_{1}\right]$ we have

$$
Q_{\varrho}^{(\kappa)}\left(z_{o}\right) \subseteq Q_{R_{2}} \subseteq Q_{2 R}
$$

For the following argument, we restrict ourselves to levels $\lambda$ with

$$
\lambda>B \lambda_{o}, \quad \text { where } \quad B:=\left(\frac{4 \hat{c} R}{R_{2}-R_{1}}\right)^{\frac{d(n+2)(1+m)}{(2 m)^{2}}}>1,
$$

and where $\hat{c}=\hat{c}(n, m)$ is the constant from the Vitali-type covering Lemma 7.1. We fix $z_{o} \in \boldsymbol{E}\left(R_{1}, \lambda\right)$ and abbreviate $\theta_{s} \equiv \theta_{z_{o} ; s}$ for $s \in(0, R]$ throughout this section. By definition of $\boldsymbol{E}\left(R_{1}, \lambda\right)$, we have

$$
\liminf _{s \downarrow 0} \iint_{Q_{s}^{\left(\theta_{s}\right)}\left(z_{o}\right)}\left[\left|D \boldsymbol{u}^{m}\right|^{2}+|F|^{2}\right] \mathrm{d} x \mathrm{~d} t \geq\left|D \boldsymbol{u}^{m}\right|^{2}\left(z_{o}\right)>\lambda^{2 m} .
$$

On the other hand, for any radius $s$ with

$$
\frac{R_{2}-R_{1}}{\hat{c}} \leq s \leq R
$$

the definition of $\lambda_{o}$, estimate (7.9), assumption (7.17) and the definition of $d$ imply

$$
\begin{aligned}
\int_{Q_{s}^{\left(\theta_{s}\right)}\left(z_{o}\right)} & {\left[\left|D \boldsymbol{u}^{m}\right|^{2}+|F|^{2}\right] \mathrm{d} x \mathrm{~d} t } \\
& \leq \frac{\left|Q_{4 R}\right|}{\left|Q_{s}^{\left(\theta_{s}\right)}\right|} \iint_{Q_{4 R}}\left[\left|D \boldsymbol{u}^{m}\right|^{2}+|F|^{2}\right] \mathrm{d} x \mathrm{~d} t \\
& \leq \frac{\left|Q_{4 R}\right|}{\left|Q_{s}\right|} \theta_{s}^{\frac{n m(1-m)}{1+m}} \lambda_{o}^{\frac{2 m}{d}} \\
& \leq\left(\frac{4 R}{s}\right)^{n+1+\frac{1}{m}+\frac{d}{2 m}\left(n+2+\frac{2}{m}\right) \cdot \frac{n m(1-m)}{1+m}} \lambda_{o}^{\frac{n m(1-m)}{1+m}+\frac{2 m}{d}} \\
& =\left(\frac{4 R}{s}\right)^{\frac{d(n+2)(1+m)}{2 m}} \lambda_{o}^{2 m} \\
& \leq B^{2 m} \lambda_{o}^{2 m}<\lambda^{2 m} .
\end{aligned}
$$

By the continuity of the mapping $s \mapsto \theta_{s}$ and the absolute continuity of the integral, the left-hand side of (7.18) depends continuously on $s$. Therefore, in view of (7.16) and (7.18), there exists a maximal radius $0<\varrho_{z_{o}}<\frac{R_{2}-R_{1}}{\hat{c}}$ for which the above inequality becomes an equality, i.e. $\varrho_{z_{o}}$ is the maximal radius with

$$
\int_{Q_{\varrho_{z_{o}}^{\left(\theta_{z_{o}}\right)}\left(z_{o}\right)}}\left[\left|D \boldsymbol{u}^{m}\right|^{2}+|F|^{2}\right] \mathrm{d} x \mathrm{~d} t=\lambda^{2 m} .
$$


The maximality of the radius $\varrho_{z_{o}}$ implies in particular that

$$
\int_{Q_{s}^{\left(\theta_{s}\right)}\left(z_{o}\right)}\left[\left|D \boldsymbol{u}^{m}\right|^{2}+|F|^{2}\right] \mathrm{d} x \mathrm{~d} t<\lambda^{2 m} \quad \text { for any } s \in\left(\varrho_{z_{o}}, R\right] .
$$

Due to the monotonicity of the mapping $\varrho \mapsto \theta_{\varrho}$ and (7.8) we have

$$
\theta_{\sigma} \leq \theta_{s} \leq\left(\frac{\sigma}{s}\right)^{\frac{d}{2 m}\left(n+2+\frac{2}{m}\right)} \theta_{\sigma} \quad \text { for any } \varrho_{z_{o}} \leq s<\sigma \leq R
$$

so that

$$
\begin{aligned}
\int_{Q_{\sigma}^{\left(\theta_{s}\right)}\left(z_{o}\right)}\left[\left|D \boldsymbol{u}^{m}\right|^{2}+|F|^{2}\right] \mathrm{d} x \mathrm{~d} t & \leq\left(\frac{\theta_{s}}{\theta_{\sigma}}\right)^{\frac{n m(1-m)}{1+m}} \iint_{Q_{\sigma}^{\left(\theta_{\sigma}\right)}\left(z_{o}\right)}\left[\left|D \boldsymbol{u}^{m}\right|^{2}+|F|^{2}\right] \mathrm{d} x \mathrm{~d} t \\
& <\left(\frac{\sigma}{s}\right)^{\frac{d n(1-m)}{2(1+m)}\left(n+2+\frac{2}{m}\right)} \lambda^{2 m}
\end{aligned}
$$

for any $\varrho_{z_{o}} \leq s<\sigma \leq R$. Finally, we recall that the cylinders are constructed in such a way that

$$
Q_{\hat{c} \varrho_{z_{o}}}^{\left(\theta_{z_{o}}\right)}\left(z_{o}\right) \subseteq Q_{\hat{c} \varrho_{z_{o}}}\left(z_{o}\right) \subseteq Q_{R_{2}}
$$

7.4. A Reverse Hölder Inequality. For a level $\lambda$ as in (7.15) and a point $z_{o} \in \boldsymbol{E}\left(R_{1}, \lambda\right)$, we consider the radius $\widetilde{\varrho}_{z_{o}} \in\left[\varrho_{z_{o}}, R\right]$ as defined in (7.7). In the sequel we write $\theta_{\varrho_{z_{o}}}$ instead of $\theta_{z_{o} ; \varrho_{z_{o}}}$. We recall that $\widetilde{\varrho}_{z_{o}}$ has been defined in such a way that for any $s \in\left[\varrho_{z_{o}}, \widetilde{\varrho}_{z_{o}}\right]$ we have $\theta_{s}=\theta_{\varrho_{z_{o}}}$, and, in particular, $\theta_{\widetilde{\varrho}_{z_{o}}}=\theta_{\varrho_{z_{o}}}$.

The aim of this section is the proof of a reverse Hölder inequality on $Q_{2 \varrho_{z_{o}}}^{\left(\theta_{\varrho_{z_{o}}}\right)}\left(z_{o}\right)$. To this end, we need to verify the assumptions of Proposition 6.1. First, we note that (7.6) with $s=4 \varrho_{z_{o}}$ implies

$$
\int_{Q_{4 \varrho z_{o}}^{\left(\theta_{\varrho} z_{o}\right)}\left(z_{o}\right)} \frac{|u|^{1+m}}{\left(4 \varrho_{z_{o}}\right)^{\frac{1+m}{m}}} \mathrm{~d} x \mathrm{~d} t \leq \theta_{\varrho_{z_{o}}}^{2 m}
$$

which means that assumption (6.1) is fulfilled for the cylinder $Q_{2 \varrho_{z_{o}}}^{\left(\theta_{\varrho_{z_{o}}}\right)}\left(z_{o}\right)$ with $K=1$. For the estimate of $\theta_{\varrho_{z_{o}}}^{2 m}$ from above, we distinguish between the cases $\widetilde{\varrho}_{z_{o}} \leq 2 \varrho_{z_{o}}$ and $\widetilde{\varrho}_{z_{o}}>2 \varrho_{z_{o}}$. In the former case, we use the fact $\theta_{\varrho_{z_{o}}}=\theta_{\widetilde{\varrho}_{z_{o}}}=\widetilde{\theta}_{\widetilde{\varrho}_{z_{o}}}$, which implies that $Q_{\widetilde{\varrho}_{z_{o}}}^{\left(\theta_{\varrho_{z_{o}}}\right)}\left(z_{o}\right)$ is intrinsic, and then the bound $\widetilde{\varrho}_{z_{o}} \leq 2 \varrho_{z_{o}}$, with the result that

$$
\theta_{\varrho_{z_{o}}}^{2 m}=\iint_{Q_{\widetilde{\varrho}_{z_{o}}}^{\left(\theta_{\left.\varrho_{z_{o}}\right)}\right)}} \frac{\left.|u|^{1+m}\right)}{\widetilde{\varrho}_{z_{o}}^{\frac{1+m}{m}}} \mathrm{~d} x \mathrm{~d} t \leq 2^{n+2+\frac{2}{m}} \iint_{Q_{2 \varrho z_{o}}^{\left(\theta_{\varrho} z_{o}\right)}\left(z_{o}\right)} \frac{|u|^{1+m}}{\left(2 \varrho_{z_{o}}\right)^{\frac{1+m}{m}}} \mathrm{~d} x \mathrm{~d} t .
$$

This means that in this case, assumption (6.2) 1 is satisfied with $K \equiv 2^{n+2+\frac{2}{m}}$. Next, we consider the remaining case $\widetilde{\varrho}_{z_{o}}>2 \varrho_{z_{o}}$. Here, we claim that

$$
\theta_{\varrho_{z_{o}}} \leq c(n, m, \nu, L) \lambda \text {. }
$$

For the proof we treat the cases $\widetilde{\varrho}_{z_{o}} \in\left(2 \varrho_{z_{o}}, \frac{R}{2}\right]$ and $\widetilde{\varrho}_{z_{o}} \in\left(\frac{R}{2}, R\right]$ separately. In the latter case, we use (7.9) with $\varrho=\widetilde{\varrho}_{z_{o}}$ and the bound $\widetilde{\varrho}_{z_{o}}>\frac{R}{2}$ in order to estimate $\theta_{\varrho_{z_{o}}}=$ $\theta_{\widetilde{\varrho}_{z_{o}}} \leq c \lambda_{o} \leq c \lambda$, which yields (7.21). In the alternative case $\widetilde{\varrho}_{z_{o}} \in\left(2 \varrho_{z_{o}}, \frac{R}{2}\right]$, the cylinder $Q_{\widetilde{\varrho}_{z_{o}}}^{\left(\theta_{\varrho_{z_{o}}}\right)}\left(z_{o}\right)$ is intrinsic by definition of $\widetilde{\varrho}_{z_{o}}$, and the two times enlarged cylinder $Q_{2 \widetilde{\varrho}_{z_{o}}}^{\left(\theta_{\varrho_{o}}\right)}\left(z_{o}\right)$ is sub-intrinsic by (7.6). Therefore, assumptions (6.1) and (6.2) 1 of Lemma 6.2 are satisfied for $Q_{\widetilde{\varrho}_{z_{o}}}^{\left(\theta_{\varrho_{z_{o}}}\right)}\left(z_{o}\right)$. The application of the lemma yields

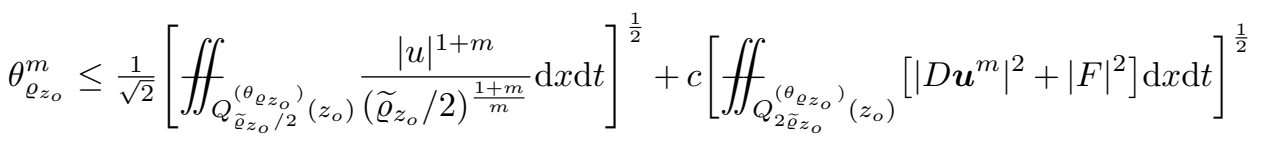


with a constant $c=c(n, m, L)$. For the first term, we exploit the sub-intrinsic coupling (7.6) with radii $\varrho=\varrho_{z_{o}}$ and $s=\frac{1}{2} \widetilde{\varrho}_{z_{o}}>\varrho_{z_{o}}$. For the estimate of the last integral, we recall that $\theta_{\widetilde{\varrho}_{z_{o}}}=\theta_{\varrho_{z_{o}}}$, which allows to use (7.20) with $s=\widetilde{\varrho}_{z_{o}}$ and $\sigma=2 \widetilde{\varrho}_{z_{o}} \in(s, R]$. This leads to the upper bound

$$
\theta_{\varrho_{z_{o}}}^{m} \leq \frac{1}{\sqrt{2}} \theta_{\varrho_{z_{o}}}^{m}+c \lambda^{m}
$$

Here, we re-absorb $\frac{1}{\sqrt{2}} \theta_{\varrho_{z_{o}}}^{m}$ into the left and obtain the claim (7.21) in any case.

Combining this with the identity (7.19), we obtain the bound

$$
\theta_{\varrho_{z_{o}}}^{2 m} \leq c \lambda^{2 m} \leq c \int_{Q_{2 \varrho_{z_{o}}}^{\left(\theta _ { \varrho _ { z _ { o } } ) } \left(_{\left.z_{o}\right)}\right.\right.}}\left[\left|D \boldsymbol{u}^{m}\right|^{2}+|F|^{2}\right] \mathrm{d} x \mathrm{~d} t .
$$

This means that in the case $\widetilde{\varrho}_{z_{o}}>2 \varrho_{z_{o}}$ assumption (6.2) 2 is satisfied on $Q_{2 \varrho_{z_{o}}}^{\left(\theta_{\varrho_{z_{o}}}\right)}$ with a constant $K \equiv K(n, m, \nu, L)$. In conclusion, in any case we have shown that all hypotheses of Proposition 6.1 are satisfied. Consequently, the proposition yields the desired reverse Hölder inequality

$$
\begin{aligned}
& \int_{\int_{2 \varrho z_{o}}^{\left(\theta_{\varrho z_{o}}\right)}\left(z_{o}\right)}\left|D \boldsymbol{u}^{m}\right|^{2} \mathrm{~d} x \mathrm{~d} t \\
& \leq c\left[\iint_{Q_{4 \varrho_{z_{o}}}^{\left(\theta_{\left.\varrho_{z_{o}}\right)}\left(z_{o}\right)\right.}}\left|D \boldsymbol{u}^{m}\right|^{2 q} \mathrm{~d} x \mathrm{~d} t\right]^{\frac{1}{q}}+c \int_{Q_{\left.4 \varrho_{z_{o}}\right)}^{\left(\theta_{\varrho}{ }_{\left(z_{o}\right)}\right.}}|F|^{2} \mathrm{~d} x \mathrm{~d} t,
\end{aligned}
$$

for a constant $c=c(n, m, \nu, L)$ and with exponent $q=\max \left\{\frac{n(1+m)}{2(n m+1+m)}, \frac{1}{2}\right\}<1$.

7.5. Estimate on super-level sets. So far we have shown that for every $\lambda$ as in (7.15) and every $z_{o} \in \boldsymbol{E}\left(R_{1}, \lambda\right)$, there exists a cylinder $Q_{\varrho_{z_{o}}}^{\left(\theta_{z_{o} \varrho_{z_{o}}}\right)}\left(z_{o}\right)$ with $Q_{\hat{c} \varrho_{z_{o}}}^{\left(\theta_{z_{o} ; \varrho_{o}}\right)}\left(z_{o}\right) \subseteq Q_{R_{2}}$, for which the properties (7.19) and (7.20), and the reverse Hölder type estimate (7.22) are satisfied. This allows us to establish a reverse Hölder inequality for the distribution function of $\left|D \boldsymbol{u}^{m}\right|^{2}$ by a Vitali covering type argument. The precise argument is as follows. We define the super-level set of the inhomogeneity $F$ by

$$
\boldsymbol{F}(r, \lambda):=\left\{z \in Q_{r}: z \text { is a Lebesgue point of } F \text { and }|F|>\lambda^{m}\right\},
$$

where again, the Lebesgue points have to be understood with respect to the cylinders constructed in Section 7.1. Using (7.19) and (7.22), we estimate

$$
\begin{aligned}
& \lambda^{2 m}=\iint_{Q_{\varrho z_{o}}^{\left(\theta_{\varrho z_{o}}\right)}\left(z_{o}\right)}\left[\left|D \boldsymbol{u}^{m}\right|^{2}+|F|^{2}\right] \mathrm{d} x \mathrm{~d} t \\
& \leq c\left[\int_{Q_{\left.4 \varrho_{z_{o}}\right)}^{\left(\theta_{\left.\varrho_{z_{0}}\right)}\left(z_{o}\right)\right.}}\left|D \boldsymbol{u}^{m}\right|^{2 q} \mathrm{~d} x \mathrm{~d} t\right]^{\frac{1}{q}}+c \int_{Q_{4 \varrho_{z_{o}}}^{\left(\theta_{\left.\varrho_{z_{o}}\right)}\left(z_{o}\right)\right.}}|F|^{2} \mathrm{~d} x \mathrm{~d} t \\
& \leq c \eta^{2 m} \lambda^{2 m}+c\left[\frac{1}{\left|Q_{4 \varrho_{z_{o}}}^{\left(\theta_{\varrho}\right)}\left(z_{o}\right)\right|} \iint_{Q_{4 \varrho z_{o}}^{\left(\theta_{\varrho_{o}}\right)}\left(z_{o}\right) \cap \boldsymbol{E}\left(R_{2}, \eta \lambda\right)}\left|D \boldsymbol{u}^{m}\right|^{2 q} \mathrm{~d} x \mathrm{~d} t\right]^{\frac{1}{q}} \\
& +\frac{c}{\left|Q_{4 \varrho_{z_{o}}}^{\left(\theta_{\varrho_{z_{o}}}\right)}\left(z_{o}\right)\right|} \iint_{Q_{4 \varrho_{z_{o}}}^{\left(\theta_{\varrho} z_{o}\right)}\left(z_{o}\right) \cap \boldsymbol{F}\left(R_{2}, \eta \lambda\right)}|F|^{2} \mathrm{~d} x \mathrm{~d} t
\end{aligned}
$$

for a constant $c=c(n, m, \nu, L)$ and any $\eta \in(0,1)$. We choose the parameter $\eta \in(0,1)$ in dependence on $n, m, \nu$, and $L$ in such a way that $\eta^{2 m}=\frac{1}{2 c}$. This allows us to re-absorb the term $\frac{1}{2} \lambda^{2 m}$ into the left-hand side. For the estimate of the second last term, we apply 
Hölder's inequality and (7.20), with the result

$$
\begin{aligned}
{\left[\frac{1}{\left|Q_{4 \varrho_{z_{o}}}^{\left(\theta_{\varrho}\right)}\left(z_{o}\right)\right|}\right.} & \left.\iint_{Q_{4 \varrho z_{o}}^{\left(\theta \varrho_{o}\right)}\left(z_{o}\right) \cap \boldsymbol{E}\left(R_{2}, \eta \lambda\right)}\left|D \boldsymbol{u}^{m}\right|^{2 q} \mathrm{~d} x \mathrm{~d} t\right]^{\frac{1}{q}-1} \\
& \leq\left[\int_{Q_{4 \varrho z_{o}}^{\left(\theta \varrho_{z_{o}}\right)}\left(z_{o}\right)}\left|D \boldsymbol{u}^{m}\right|^{2} \mathrm{~d} x \mathrm{~d} t\right]^{1-q} \leq c \lambda^{2 m(1-q)}
\end{aligned}
$$

We use this to estimate the right-hand side of (7.23). The resulting inequality is then multiplied by $\left|Q_{\left.4 \varrho_{z_{o}}\right)}^{\left(\theta_{\varrho z_{0}}\right)}\left(z_{o}\right)\right|$. In this way, we arrive at

$$
\begin{aligned}
\lambda^{2 m}\left|Q_{4 \varrho_{z_{o}}}^{\left(\theta_{\varrho z_{o}}\right)}\left(z_{o}\right)\right| \leq & c \iint_{Q_{4 \varrho z_{o}}^{\left(\theta \theta_{z_{o}}\right)}\left(z_{o}\right) \cap \boldsymbol{E}\left(R_{2}, \eta \lambda\right)} \lambda^{2 m(1-q)}\left|D \boldsymbol{u}^{m}\right|^{2 q} \mathrm{~d} x \mathrm{~d} t \\
& +c \iint_{Q_{4 \varrho z_{o}}^{\left(\theta e_{z_{o}}\right)}\left(z_{o}\right) \cap \boldsymbol{F}\left(R_{2}, \eta \lambda\right)}|F|^{2} \mathrm{~d} x \mathrm{~d} t,
\end{aligned}
$$

where $c=c(n, m, \nu, L)$. On the other hand, we bound the left-hand side from below by the use of (7.20). This leads to the inequality

$$
\lambda^{2 m} \geq c \int_{\int_{\hat{c} \varrho z_{o}}^{\left(\theta \varrho z_{o}\right)}\left(z_{o}\right)}\left|D \boldsymbol{u}^{m}\right|^{2} \mathrm{~d} x \mathrm{~d} t,
$$

where we relied on the fact that $\hat{c}$ is a universal constant depending only on $n$ and $m$. Combining the two preceding estimates and using again that $\hat{c}=\hat{c}(n, m)$, we arrive at

$$
\begin{aligned}
\iint_{Q_{\hat{c} \varrho z_{o}}^{\left(\theta \varrho_{z_{o}}\right)}\left(z_{o}\right)}\left|D \boldsymbol{u}^{m}\right|^{2} \mathrm{~d} x \mathrm{~d} t \leq & c \iint_{Q_{4 \varrho z_{o}}^{\left(\theta_{\varrho} z_{o}\right)}\left(z_{o}\right) \cap \boldsymbol{E}\left(R_{2}, \eta \lambda\right)} \lambda^{2 m(1-q)}\left|D \boldsymbol{u}^{m}\right|^{2 q} \mathrm{~d} x \mathrm{~d} t \\
& +c \iint_{Q_{4 \varrho z_{o}}^{\left(\theta_{\varrho} e_{z_{o}}\right)}\left(z_{o}\right) \cap \boldsymbol{F}\left(R_{2}, \eta \lambda\right)}|F|^{2} \mathrm{~d} x \mathrm{~d} t
\end{aligned}
$$

for a constant $c=c(n, m, \nu, L)$. Since the preceding inequality holds for every center $z_{o} \in \boldsymbol{E}\left(R_{1}, \lambda\right)$, we conclude that it is possible to cover the super-level set $\boldsymbol{E}\left(R_{1}, \lambda\right)$ by a family $\mathcal{F} \equiv\left\{Q_{4 \varrho_{z_{o}} \varrho_{o}}^{\left(\theta_{z_{0}}\right)}\left(z_{o}\right)\right\}$ of parabolic cylinders with center $z_{o} \in \boldsymbol{E}\left(R_{1}, \lambda\right)$, such that each of the cylinders is contained in $Q_{R_{2}}$, and that on each cylinder estimate (7.24) is valid. An application of the Vitali type Covering Lemma 7.1 provides us with a countable disjoint subfamily

with the property

$$
\left\{Q_{4 \varrho_{z_{i}}}^{\left(\theta_{z_{i}, \varrho_{z_{i}}}\right)}\left(z_{i}\right)\right\}_{i \in \mathbb{N}} \subseteq \mathcal{F}
$$

$$
\boldsymbol{E}\left(R_{1}, \lambda\right) \subseteq \bigcup_{i=1}^{\infty} Q_{\hat{c} \varrho_{z_{i}}}^{\left(\theta_{z_{i} ; \varrho_{z_{i}}}\right)}\left(z_{i}\right) \subseteq Q_{R_{2}}
$$

We apply (7.24) for each of the cylinders $Q_{4 \varrho_{z_{i}}}^{\left(\theta_{z_{i} ; \varrho z_{i}}\right)}\left(z_{i}\right)$ and add the resulting inequalities. Since the cylinders $Q_{4 \varrho_{z_{i}}}^{\left(\theta_{z_{i} ; \varrho_{i}}\right)}\left(z_{i}\right)$ are pairwise disjoint, we obtain

$\iint_{\boldsymbol{E}\left(R_{1}, \lambda\right)}\left|D \boldsymbol{u}^{m}\right|^{2} \mathrm{~d} x \mathrm{~d} t \leq c \iint_{\boldsymbol{E}\left(R_{2}, \eta \lambda\right)} \lambda^{2 m(1-q)}\left|D \boldsymbol{u}^{m}\right|^{2 q} \mathrm{~d} x \mathrm{~d} t+c \iint_{\boldsymbol{F}\left(R_{2}, \eta \lambda\right)}|F|^{2} \mathrm{~d} x \mathrm{~d} t$, with $c=c(n, m, \nu, L)$. In order to compensate for the fact that super-level sets of different levels appear on both sides of the preceeding estimate, we need an estimate on the difference $\boldsymbol{E}\left(R_{1}, \eta \lambda\right) \backslash \boldsymbol{E}\left(R_{1}, \lambda\right)$. However, on this set we can simply estimate $\left|D \boldsymbol{u}^{m}\right|^{2} \leq \lambda^{2 m}$, which leads to the bound

$$
\iint_{\boldsymbol{E}\left(R_{1}, \eta \lambda\right) \backslash \boldsymbol{E}\left(R_{1}, \lambda\right)}\left|D \boldsymbol{u}^{m}\right|^{2} \mathrm{~d} x \mathrm{~d} t \leq \iint_{\boldsymbol{E}\left(R_{2}, \eta \lambda\right)} \lambda^{2 m(1-q)}\left|D \boldsymbol{u}^{m}\right|^{2 q} \mathrm{~d} x \mathrm{~d} t .
$$


Adding the last two inequalities, we obtain a reverse Hölder type inequality for the distribution function of $\left|D \boldsymbol{u}^{m}\right|^{2}$ for levels $\eta \lambda$. In this inequality we replace $\eta \lambda$ by $\lambda$ and recall that $\eta \in(0,1)$ was chosen as a universal constant depending only on $n, m, \nu$, and $L$. In this way, we obtain for any $\lambda \geq \eta B \lambda_{o}=: \lambda_{1}$ that

$$
\begin{aligned}
\iint_{\boldsymbol{E}\left(R_{1}, \lambda\right)} \mid & \left.D \boldsymbol{u}^{m}\right|^{2} \mathrm{~d} x \mathrm{~d} t \\
& \leq c \iint_{\boldsymbol{E}\left(R_{2}, \lambda\right)} \lambda^{2 m(1-q)}\left|D \boldsymbol{u}^{m}\right|^{2 q} \mathrm{~d} x \mathrm{~d} t+c \iint_{\boldsymbol{F}\left(R_{2}, \lambda\right)}|F|^{2} \mathrm{~d} x \mathrm{~d} t
\end{aligned}
$$

with a constant $c=c(n, m, \nu, L)$. This is the desired reverse Hölder type inequality for the distribution function of $D \boldsymbol{u}^{m}$.

7.6. Proof of the gradient estimate. Once (7.25) is established the final higher integrability result follows by integrating (7.25) over the range of possible values $\lambda$. Here, one has to take into account that the existence of certain integrals appearing in the proof are not guaranteed in advance. This technical point can be overcome by use of truncation methods. Since all arguments have been elaborated in detail for example in [3, 2] we omit the details, and only state the final outcome. There exists $\varepsilon_{o}=\varepsilon_{o}(n, m, \nu, L) \in(0,1]$ such that for any $0<\varepsilon<\varepsilon_{1}:=\min \left\{\varepsilon_{o}, \sigma-2\right\}$ we have

$$
\int_{Q_{R}}\left|D \boldsymbol{u}^{m}\right|^{2+\varepsilon} \mathrm{d} x \mathrm{~d} t \leq c \lambda_{o}^{\varepsilon m} \int_{Q_{2 R}}\left|D \boldsymbol{u}^{m}\right|^{2} \mathrm{~d} x \mathrm{~d} t+c \int_{Q_{2 R}}|F|^{2+\varepsilon} \mathrm{d} x \mathrm{~d} t .
$$

At this stage it remains to bound $\lambda_{o}$. This can be achieved by an application of the energy estimate from Lemma 4.1 with $\theta=1$ and $a=0$ and Young's inequality. Indeed, we have

$$
\lambda_{o} \leq c\left[1+\iint_{Q_{8 R}}\left[\frac{|u|^{1+m}}{R^{\frac{1+m}{m}}}+|F|^{2}\right] \mathrm{d} x \mathrm{~d} t\right]^{\frac{d}{2 m}}
$$

where $c=c(n, m, \nu, L)$. Plugging this into the preceding estimate, we arrive at

$$
\begin{aligned}
\int_{Q_{R}}\left|D \boldsymbol{u}^{m}\right|^{2+\varepsilon} \mathrm{d} x \mathrm{~d} t \leq c[1+ & \left.\int_{Q_{8 R}}\left[\frac{|u|^{1+m}}{R^{\frac{1+m}{m}}}+|F|^{2}\right] \mathrm{d} x \mathrm{~d} t\right]^{\frac{\varepsilon d}{2}} \oiiint_{Q_{2 R}}\left|D \boldsymbol{u}^{m}\right|^{2} \mathrm{~d} x \mathrm{~d} t \\
& +c \int_{Q_{2 R}}|F|^{2+\varepsilon} \mathrm{d} x \mathrm{~d} t
\end{aligned}
$$

for a constant $c=c(n, m, \nu, L)$. The asserted reverse Hölder inequality (2.6) now follows by a standard covering argument. More precisely, we cover $Q_{R}$ by finitely many cylinders of radius $\frac{R}{8}$, apply the preceding estimate on each of the smaller cylinders and take the sum. This yields the same estimate as above, but with integrals over $Q_{2 R}$ instead of $Q_{8 R}$. This completes the proof of Theorem 2.2.

7.7. Proof of Corollary 2.5. We consider a standard parabolic cylinder $C_{2 R}\left(z_{o}\right):=$ $B_{2 R}\left(x_{o}\right) \times\left(t_{o}-(2 R)^{2}, t_{o}+(2 R)^{2}\right) \subseteq \Omega_{T}$ and rescale the problem via

$$
\left\{\begin{array}{c}
v(x, t):=u\left(x_{o}+R x, t_{o}+R^{2} t\right) \\
\mathbf{B}(x, t, u, \xi):=R \mathbf{A}\left(x_{o}+R x, t_{o}+R^{2} t, u, \frac{1}{R} \xi\right) \\
G(x, t):=R F\left(x_{o}+R x, t_{o}+R^{2} t\right),
\end{array}\right.
$$

whenever $(x, t) \in C_{2}$ and $(u, \xi) \in \mathbb{R}^{N} \times \mathbb{R}^{N n}$. The rescaled function $v$ is a weak solution of the differential equation

$$
\partial_{t} v-\operatorname{div} \mathbf{B}\left(x, t, v, D \boldsymbol{v}^{m}\right)=\operatorname{div} G \quad \text { in } \widetilde{Q}:=Q_{2^{\frac{2 m}{1+m}}} \subseteq C_{2}
$$


in the sense of Definition 2.1. Moreover, the rescaled vector-field B satisfies assumptions (2.3). Consequently, we can apply estimate (2.6) to $v$ on the cylinder $\widetilde{Q}$, which gives

$$
\begin{aligned}
& \int_{\frac{1}{2} \widetilde{Q}}\left|D \boldsymbol{v}^{m}\right|^{2+\varepsilon} \mathrm{d} x \mathrm{~d} t \\
& \quad \leq c\left[1+\iint_{\widetilde{Q}}\left[|v|^{1+m}+|G|^{2}\right] \mathrm{d} x \mathrm{~d} t\right]^{\frac{\varepsilon d}{2}} \iint_{\widetilde{Q}}\left|D \boldsymbol{v}^{m}\right|^{2} \mathrm{~d} x \mathrm{~d} t+c \int_{\widetilde{Q}}|G|^{2+\varepsilon} \mathrm{d} x \mathrm{~d} t,
\end{aligned}
$$

for every $\varepsilon \in\left(0, \varepsilon_{1}\right]$, where $c=c(n, m, \nu, L)$, and we abbreviated $\frac{1}{2} \widetilde{Q}:=Q_{2^{\frac{m-1}{1+m}}}$. Scaling back and using the fact that the cylinder $C_{\gamma R}$ with $\gamma:=2^{\frac{m-1}{2 m}}$ is contained in the re-scaled version of the cylinder $\frac{1}{2} \widetilde{Q}$ we deduce

$$
\begin{aligned}
& R^{2+\varepsilon} \iint_{C_{\gamma R}\left(z_{o}\right)}\left|D \boldsymbol{u}^{m}\right|^{2+\varepsilon} \mathrm{d} x \mathrm{~d} t \\
& \quad \leq c R^{2}\left[1+\int_{C_{2 R}\left(z_{o}\right)}\left[|u|^{1+m}+R^{2}|F|^{2}\right] \mathrm{d} x \mathrm{~d} t\right]^{\frac{\varepsilon d}{2}} \iint_{C_{2 R}\left(z_{o}\right)}\left|D \boldsymbol{u}^{m}\right|^{2} \mathrm{~d} x \mathrm{~d} t \\
& \quad+c R^{2+\varepsilon} \int_{C_{2 R}\left(z_{o}\right)}|F|^{2+\varepsilon} \mathrm{d} x \mathrm{~d} t .
\end{aligned}
$$

The asserted estimate on the pair $C_{R}, C_{2 R}$ of standard parabolic cylinders now follows with a standard covering argument. This finishes the proof of Corollary 2.5.

\section{REFERENCES}

[1] V. Bögelein. Higher integrability for weak solutions of higher order degenerate parabolic systems. Ann. Acad. Sci. Fenn. Math., 33 (2008), no. 2, 387-412.

[2] V. Bögelein, F. Duzaar, J. Kinnunen, and C. Scheven. The higher integrability of weak solutions doubly nonlinear parabolic systems. Preprint 2018, arXiv:1810.06039.

[3] V. Bögelein, F. Duzaar, R. Korte, and C. Scheven. The higher integrability of weak solutions of porous medium systems. Adv. Nonlinear Anal., DOI: https://doi.org/10.1515/anona-2017-0270.

[4] V. Bögelein and M. Parviainen. Self-improving property of nonlinear higher order parabolic systems near the boundary. NoDEA Nonlinear Differential Equations Appl., 17 (2010), no. 1, 21-54.

[5] L.A. Caffarelli, J. L. Vázquez, and N. I. Wolanski. Lipschitz continuity of solutions and interfaces of the N-dimensional porous medium equation. Indiana Univ. Math. J., 36(2) (1987), 373-401.

[6] E. DiBenedetto. Degenerate parabolic equations. Springer-Verlag, Universitytext xv, 387, New York, NY, 1993.

[7] Emmanuele DiBenedetto and Avner Friedman. Hölder estimates for nonlinear degenerate parabolic systems. J. Reine Angew. Math., 357:1-22, 1985.

[8] E. DiBenedetto, U. Gianazza, and V. Vespri. Harnack's inequality for degenerate and singular parabolic equations. Springer Monographs in Mathematics, 2011.

[9] L. Diening, P. Kaplický, and S. Schwarzacher. BMO estimates for the $p$-Laplacian, Nonlinear Anal., 75 (2012), no. 2, 637-650.

[10] H. Federer. Geometric measure theory. Die Grundlehren der mathematischen Wissenschaften, Band 153, Springer-Verlag, New York, 1969.

[11] U. Gianazza and S. Schwarzacher. Self-improving property of degenerate parabolic equations of porous medium-type. Amer. J. Math., to appear.

[12] U. Gianazza and S. Schwarzacher. Self-improving property of the fast diffusion equation. Preprint 2018, arXiv:1810.04557.

[13] M. Giaquinta. Multiple Integrals in the Calculus of Variations and Nonlinear Elliptic Systems. Princeton University Press, Princeton, 1983.

[14] M. Giaquinta and M. Struwe. On the partial regularity of weak solutions of nonlinear parabolic systems. Math. Z., 179 (1982), no. 4, 437-451.

[15] E. Giusti. Direct Methods in the Calculus of Variations. World Scientific Publishing Company, Tuck Link, Singapore, 2003.

[16] J. Kinnunen and J. L. Lewis. Higher integrability for parabolic systems of $p$-Laplacian type. Duke Math. J., 102 (2000), no. 2, 253-271.

[17] N. G. Meyers and A. Elcrat. Some results on regularity for solutions of non-linear elliptic systems and quasi-regular functions. Duke Math. J., 42 (1975), 121-136. 
V. BÖGELEIN, F. DUZAAR, AND C. SCHEVEN

[18] M. Parviainen. Global gradient estimates for degenerate parabolic equations in nonsmooth domains. Ann. Mat. Pura Appl. (4), 188 (2009), no. 2, 333-358.

[19] S. Schwarzacher. Hölder-Zygmund estimates for degenerate parabolic systems. J. Differential Equations, 256 (2014), no. 7, 2423-2448.

[20] J. Vázquez. Smoothing and decay estimates for nonlinear diffusion equations. Equations of porous medium type. Oxford Lecture Series in Mathematics and its Applications, 33. Oxford University Press, Oxford, 2006.

[21] J. Vázquez. The porous medium equation. Mathematical theory. Oxford Mathematical Monographs. The Clarendon Press, Oxford University Press, Oxford, 2007.

Verena Bögelein, Fachbereich Mathematik, Universität Salzburg, Hellbrunner Str. 34, 5020 SAlZBUrg, AUSTRIA

E-mail address: verena.boegelein@sbg.ac.at

Frank DuZaAr, Department MAThematik, Universität ERlangen-NÜRnberg, CAUERSTRASSE 11, 91058 ERLANGEN, GERMANY

E-mail address: frank.duzaarefau.de

Christoph Scheven, Fakultät Für Mathematik, Universität Duisburg-Essen, 45117 EsSEN, GERMANY

E-mail address: christoph.scheven@uni-due.de 